\title{
Pseudogap and Fermi-Surface Topology in the Two-Dimensional Hubbard Model
}

\author{
Wei Wu, ${ }^{1,2}$ Mathias S. Scheurer, ${ }^{3}$ Shubhayu Chatterjee, ${ }^{3}$ Subir Sachdev, ${ }^{3,4,5}$ Antoine Georges, ${ }^{2,6,1,7}$ and Michel Ferrero ${ }^{1,2}$ \\ ${ }^{1}$ Centre de Physique Théorique, Ecole Polytechnique, CNRS, \\ Université Paris-Saclay, 91128 Palaiseau, France \\ ${ }^{2}$ Collège de France, 11 place Marcelin Berthelot, 75005 Paris, France \\ ${ }^{3}$ Department of Physics, Harvard University, Cambridge, Massachusetts 02138, USA \\ ${ }^{4}$ Perimeter Institute for Theoretical Physics, Waterloo, Ontario, Canada N2L $2 Y 5$ \\ ${ }^{5}$ Department of Physics, Stanford University, Stanford, California 94305, USA \\ ${ }^{6}$ Center for Computational Quantum Physics, Flatiron Institute, \\ 162 Fifth Avenue, New York, New York 10010, USA \\ ${ }^{7} D Q M P$, Université de Genève, 24 quai Ernest Ansermet, CH-1211 Genève, Switzerland
}

(Received 20 July 2017; revised manuscript received 30 March 2018; published 22 May 2018; corrected 4 June 2018)

\begin{abstract}
One of the distinctive features of hole-doped cuprate superconductors is the onset of a "pseudogap" below a temperature $T^{*}$. Recent experiments suggest that there may be a connection between the existence of the pseudogap and the topology of the Fermi surface. Here, we address this issue by studying the twodimensional Hubbard model with two distinct numerical methods. We find that the pseudogap only exists when the Fermi surface is holelike and that, for a broad range of parameters, its opening is concomitant with a Fermi-surface topology change from electronlike to holelike. We identify a common link between these observations: The polelike feature of the electronic self-energy associated with the formation of the pseudogap is found to also control the degree of particle-hole asymmetry, and hence the Fermi-surface topology transition. We interpret our results in the framework of an $\mathrm{SU}(2)$ gauge theory of fluctuating antiferromagnetism. We show that a mean-field treatment of this theory in a metallic state with $U(1)$ topological order provides an explanation of this polelike feature and a good description of our numerical results. We discuss the relevance of our results to experiments on cuprates.
\end{abstract}

DOI: 10.1103/PhysRevX.8.021048

Subject Areas: Condensed Matter Physics, Strongly Correlated Materials, Superconductivity

\section{INTRODUCTION}

A very debated topic in the physics of high-temperature superconductors is the nature of the "pseudogap" [1,2] in their phase diagram. Below a temperature $T^{*}(p)$, which is a decreasing function of the hole-doping level $p$, a pseudogap develops, corresponding to a suppression of lowenergy excitations apparent in many experimental probes. Extrapolated to zero temperature, $T^{*}(p)$ defines a critical hole doping $p^{*}$ above which the pseudogap disappears as doping is increased. Another important critical value of the doping, denoted here $p_{\mathrm{FS}}$, is that at which the Fermi-surface topology changes from holelike to electronlike, corresponding to a Lifshitz transition. Recent experiments on $\mathrm{Bi}_{2} \mathrm{Sr}_{2} \mathrm{CaCu}_{2} \mathrm{O}_{8+\delta}$ (Bi2212) have suggested that the pseudogap may be very sensitive to the Fermi-surface (FS)

Published by the American Physical Society under the terms of the Creative Commons Attribution 4.0 International license. Further distribution of this work must maintain attribution to the author(s) and the published article's title, journal citation, and DOI. topology and that $p^{*} \simeq p_{\mathrm{FS}}$ in this compound $[3,4]$. In a simultaneous and independent manner from the present theoretical work, Doiron-Leyraud et al. [5] recently performed a systematic experimental study using hydrostatic pressure as a control parameter in the $\mathrm{La}_{1.6-x} \mathrm{Nd}_{0.4} \mathrm{Sr}_{x} \mathrm{CuO}_{4}$ (Nd-LSCO) system, and an unambiguous connection between FS topology and the pseudogap was found.

In this work, we investigate this interplay by studying the two-dimensional Hubbard model. In the weak-coupling scenarios of pseudogap physics, there is a natural connection between the FS topology and the coherence of lowenergy quasiparticles. Indeed, for a holelike Fermi surface, coherence is suppressed at the "hot spots," where the Fermi surface intersects the antiferromagnetic zone boundary. When the Fermi surface turns electronlike, increased quasiparticle coherence is restored all along the Fermi surface (see Appendix E for a more detailed analysis) [6-11]. At stronger coupling, several methods $[9,12-28]$ have established that the Hubbard model displays a pseudogap that originates from antiferromagnetic correlations. These correlations become short range as the coupling strength or doping level is increased, as found in experiments [29]. The FS topology, 
on the other hand, is an issue that has to do with low-energy, long-distance physics. Hence, it is an intriguing and fundamentally important question to understand how the shortrange correlations responsible for the pseudogap can be sensitive to Fermi surface changes.

Here, we study the Hubbard model for a broad range of parameters, and we analyze the pseudogap, FS topology, and their interplay. We show that, at strong coupling, interactions can strongly modify the Fermi surface, making it more holelike as compared to its noninteracting shape $[16,30-33]$. We find that a pseudogap only exists when the Fermi surface is holelike, so that $p^{*} \leq p_{\mathrm{FS}}$. We identify an extended parameter regime in which these two critical doping levels are very close to one another: $p^{*} \simeq p_{\mathrm{FS}}$, so that the Fermi surface turns electronlike only when the pseudogap collapses. Moreover we show that, when considering the relation between the pseudogap and FS topology, hole-doped cuprates can be separated into two families: materials for which $p^{*} \simeq p_{\mathrm{FS}}$ and materials which have $p^{*}<p_{\mathrm{FS}}$. These two families differ mostly by the relative magnitude of the next nearest-neighbor hopping. These findings are shown to be consistent with a large body of experiments on cuprates.

We reveal that a common link between these observations is the polelike feature $[22,23,30,32,34-36]$ displayed by the electronic self-energy at the antinodal point, $\boldsymbol{k}=(\pi, 0)$. The large imaginary part of the antinodal self-energy associated with this pole is responsible for the pseudogap, while the large particle-hole asymmetry associated with its real part controls the interaction-induced deformation of the Fermi surface and the location of the Fermi-surface topology transition. We investigate the evolution of this particle-hole asymmetry as a function of doping and nearest-neighbor hopping $t^{\prime}$, and we show that the line in $\left(p-t^{\prime}\right)$ space where particle-hole symmetry is approximately obeyed at low energy is pushed, at strong coupling, to very low values of $p$ and very negative values of $t^{\prime}$. This is in stark contrast to the results of weak-coupling theories where this line is close to the Lifshitz transition of the noninteracting system. This also explains why interactions drive the Fermi surface more holelike for hole doping.

In order to understand these results from a more analytic standpoint, we consider a recently developed $\mathrm{SU}(2)$ gauge theory of fluctuating antiferromagnetic order [37,38]; additional results on the $\mathrm{SU}(2)$ gauge theory appear in a companion paper, Ref. [39]. We focus on a metallic phase of this theory, characterized by U(1) topological order, which does not break spin or translational symmetries. We show that a mean-field treatment of this gauge theory provides a good description of our numerical results. In particular, the self-energy of the charge-carrying field (chargon) in this theory displays a pole that provides an explanation for the quasipole of the physical electron selfenergy. The latter is calculated and compares well to our numerical results, as do the trends in the evolution of the pseudogap and particle-hole asymmetry as a function of $p$ and $t^{\prime}$.

This paper is organized as follows. In Sec. II, we briefly introduce the model and the numerical methods used in this article. In Sec. III, we study the interplay between the pseudogap and FS topology and analyze the mechanisms controlling this interplay. The comparison and interpretation of our results in terms of the $\mathrm{SU}(2)$ gauge theory is presented at the end of this section. In Sec. IV, we discuss the relevance of our results to experiments on hole-doped cuprates. Section V provides a conclusion and outlook. Finally, details about the employed methods and various supporting materials can be found in the appendixes.

\section{MODEL AND METHOD}

We consider the Hubbard model defined by the Hamiltonian

$$
\mathcal{H}=-\sum_{i j, \sigma} t_{i j} c_{i, \sigma}^{\dagger} c_{j, \sigma}+U \sum_{i} n_{i \uparrow} n_{i \downarrow}-\mu \sum_{i, \sigma} n_{i \sigma}
$$

where $U$ is the on-site Coulomb repulsion and $\mu$ the chemical potential. The hopping amplitudes $t_{i j}$ 's are chosen to be nonzero between nearest-neighbor sites $\left(t_{i j}=t\right)$ and next-nearest-neighbor ones $\left(t_{i j}=t^{\prime}\right)$. These hopping amplitudes define a noninteracting dispersion relation $\epsilon_{\mathbf{k}}=-2 t\left(\cos k_{x}+\cos k_{y}\right)-4 t^{\prime} \cos k_{x} \cos k_{y}$. In the following, $t=1$ will be our unit of energy. We solve this model using two distinct methods: the dynamical cluster approximation (DCA) [15] and determinant quantum Monte Carlo (DQMC) [40]; see the Appendix A for details. Cluster extensions of dynamical mean-field theory (DMFT) have shown that the Hubbard model is able to capture many features of cuprate superconductors, such as the superconducting dome and the pseudogap [9,14-18, 20-23,26,32,41-43]. They have also established that the pseudogap originates from antiferromagnetic correlations, which become short-range as the coupling strength or doping level are increased. This was also recently corroborated by exact diagrammatic Monte Carlo simulations [28]. While cluster extensions of DMFT have shown that hole doping can drive a Lifshitz transition [30-32], no general relationship between the pseudogap and FS topology has been established. We therefore carry out a systematic study for a broad range of parameters in order to investigate this issue.

\section{RESULTS}

\section{A. Pseudogap and FS topology}

In Fig. 1, we display the pseudogap onset temperature $T^{*}(p)$ and the temperature $T_{\mathrm{FS}}(p)$, at which the Fermi surface changes its topology, as a function of doping level $p$, for several values of the next-nearest-neighbor hopping 


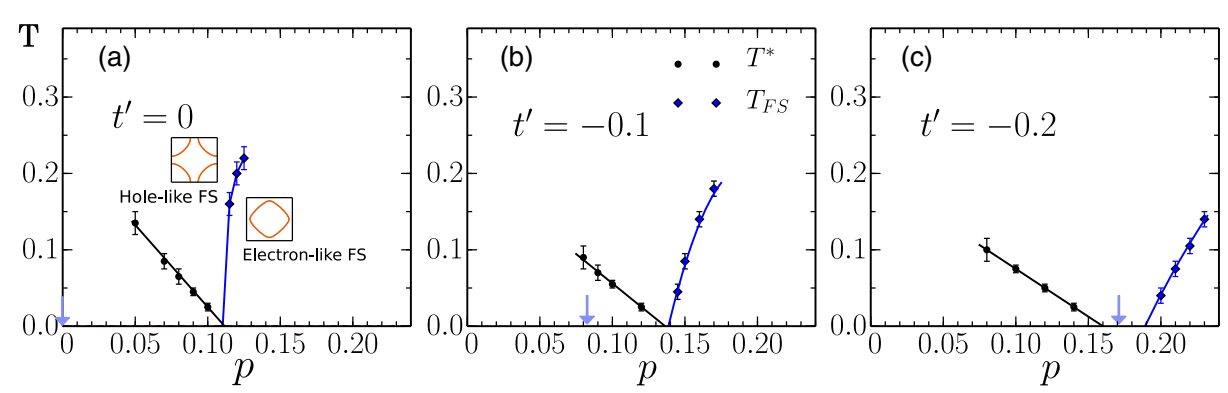

FIG. 1. Pseudogap and Lifshitz transition temperatures. Evolution of the pseudogap onset temperature $T^{*}$ (black) and the Lifshitz transition temperature $T_{\mathrm{FS}}$ (blue) as a function of the hole doping $p$, for several values of $t^{\prime}$ and $U=7$. The finite temperature data points are extrapolated to zero temperature and yield two critical dopings $p^{*}$ and $p_{\mathrm{FS}}$. It is apparent that $p^{*} \simeq p_{\mathrm{FS}}$ for $t^{\prime}=0$ and $t^{\prime}=-0.1$, while $p^{*}<p_{\mathrm{FS}}$ for $t^{\prime}=-0.2$. The solid lines are linear (for $T^{*}$ ) and quadratic (for $T_{\mathrm{FS}}$ ) least-squares fits to the data points, except the $T_{\mathrm{FS}}$ line of $t^{\prime}=0$, where $T_{\mathrm{FS}}$ collapses to zero close to $p^{*}$. Error bars estimate all uncertainties in finding $T^{*}$ and $T_{\mathrm{FS}}$ with DCA (see also Appendix B). Note that the change of topology of the Fermi surface for the interacting system occurs at a larger doping than that of the noninteracting system (indicated by a light blue arrow).

$t^{\prime} . T^{*}$ is identified as follows: We calculate the zerofrequency extrapolated value of the spectral function at the antinodal point $(\pi, 0)$; we find that its temperature dependence displays a maximum, which we identify as $T^{*}$. Below this scale, the antinodal spectral intensity decreases, signaling the opening of a pseudogap. $T_{\mathrm{FS}}$ is identified as the temperature where the Fermi surface crosses the $(\pi, 0)$ point and turns from holelike to electronlike as temperature decreases (see below). Note that our definition of a Fermi surface is a pragmatic one: strictly speaking a Fermi surface only exists at zero temperature. At finite temperatures, we define the Fermi surface as the surface in momentum space corresponding to the maximum of the spectral intensity as it would be observed, e.g., in an angle-resolved photoemission (ARPES) experiment [44].

When extrapolated to zero temperature, these data define two critical doping levels: $p^{*}$ such that the pseudogap disappears for $p>p^{*}$, and $p_{\mathrm{FS}}$ that marks the transition from a holelike FS $\left(p<p_{\mathrm{FS}}\right)$ to an electronlike FS $\left(p>p_{\mathrm{FS}}\right)$. Strikingly, the two curves in Fig. 1 suggest that the pseudogap can only exist when the Fermi surface is holelike, i.e., that $p^{*} \leq p_{\mathrm{FS}}$. It appears that, for values of $t^{\prime} \geq-0.1$, both transitions happen at the same doping $p^{*}=p_{\mathrm{FS}}$ within our error bars. For more negative values of $t^{\prime}$, the Fermi surface first becomes holelike as $p$ is reduced, and the pseudogap opens at a lower doping, i.e., $p^{*}<p_{\mathrm{FS}}$. We never observe a pseudogap with an electronlike Fermi surface, which would correspond to $p^{*}>p_{\mathrm{FS}}$.

This can be documented further by repeating this analysis for several doping levels' $p$ and $t^{\prime}$ values. The resulting map in the $\left(p-t^{\prime}\right)$ parameter space is displayed in Fig. 2. A first observation is that the topological transition of the FS (blue line) that separates the regions with holelike and electronlike Fermi surfaces is strongly renormalized with respect to its noninteracting $(U=0)$ location (dashed line in Fig. 2 and arrows in Fig. 1). The black line defines the onset of the pseudogap. These lines define three regions: At large doping above the blue line, the Fermi surface is electronlike and no pseudogap is present. In the intermediate region between the two lines, the Fermi surface is holelike but without a pseudogap. The topological transition and pseudogap opening coincide for a range of $t^{\prime}$, while, for more negative $t^{\prime}$, the two lines split apart and, as doping is reduced, the pseudogap only opens after the Fermi surface has already turned holelike at higher doping level $\left(p^{*}<p_{\mathrm{FS}}\right)$. The pseudogap and FS topology transition lines are dependent on the value of $U$. As detailed in the Appendix $\mathrm{B}$, a larger value of $U$ yields a more

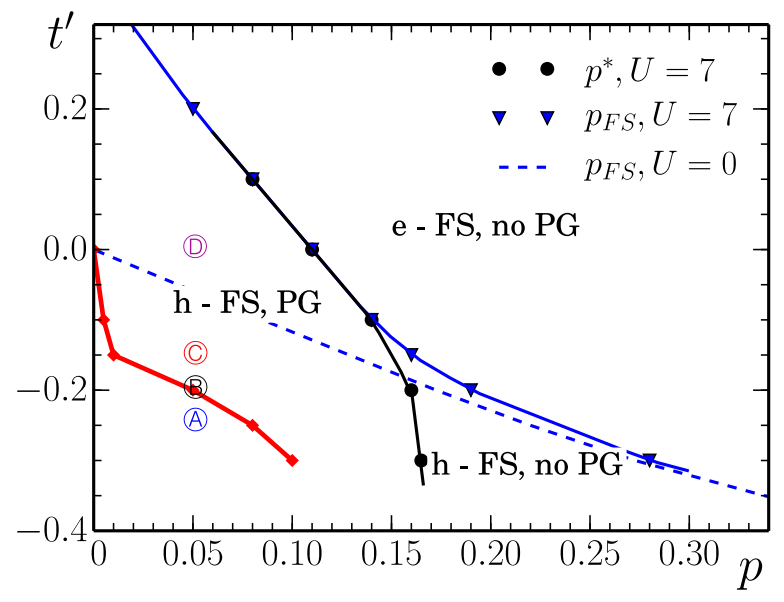

FIG. 2. Zero-temperature Fermi-surface topology and pseudogap in the $p-t^{\prime}$ plane. The black line separates a region with no pseudogap (no PG) from a region where a pseudogap exists (PG). The blue line indicates where the interacting Fermi surface changes its topology from electronlike (e-FS) to holelike (h-FS). The dashed blue line signals the same transition in the noninteracting case. The red curve locates the change in particle-hole asymmetry at the antinode: above the red line the real part of the self-energy modifies the Fermi surface towards a more holelike shape. On the red line, the self-energy pole crosses zero energy and approximate particlehole symmetry is restored, corresponding also to a maximum of the low-energy scattering rate as $t^{\prime}$ is varied for fixed $p$. Points $A-D$ label a set of parameters that are discussed further in Fig. 4. 
extended regime of parameters for which $p^{*} \simeq p_{\mathrm{FS}}$, with the "branching point" where the two lines merge moving towards more negative values of $t^{\prime}$ and a larger doping level. This observation is important when comparing to experimental observations (see below).

\section{B. Change of Fermi-surface topology due to correlation effects}

The Fermi-surface topology at the antinode is controlled by the renormalized quasiparticle energy,

$$
\begin{aligned}
\tilde{\epsilon}_{(\pi, 0)} & =\epsilon_{(\pi, 0)}-\mu+\operatorname{Re} \Sigma_{(\pi, 0)}(\omega=0) \\
& =4 t^{\prime}-\mu+\operatorname{Re} \Sigma_{(\pi, 0)}(\omega=0) .
\end{aligned}
$$

For negative values of $\tilde{\epsilon}_{(\pi, 0)}$, the Fermi surface is holelike, while it is electronlike for $\tilde{\epsilon}_{(\pi, 0)}>0$. In order to gain insight into the mechanisms driving the Lifshitz transition, Fig. 3 displays $\tilde{\epsilon}_{(\pi, 0)}$ as a function of temperature for various doping levels, with arrows indicating $T^{*}$ and $T_{\mathrm{FS}}$. Interestingly, even at the highest temperature $T=0.2$ displayed there, $\tilde{\epsilon}_{(\pi, 0)}$ is negative for all doping levels, yielding a holelike Fermi surface, while the noninteracting Fermi surface would be electronlike for $p \gtrsim 9 \%$. This temperature is above the pseudogap temperature $T^{*}$ and, hence, the renormalization of the Fermi surface would be visible on a full Fermi surface in an ARPES experiment. In this high-temperature range, only local correlations are responsible for this effect, as already captured in a singlesite DMFT calculation (see Fig. 12 in the Appendix C). As the temperature is decreased, $\tilde{\epsilon}_{(\pi, 0)}$ first increases slightly but then suddenly drops to very negative values, pushing the Fermi surface to be very holelike at low temperatures. This starts happening just above the pseudogap temperature, and both effects can be traced back to nonlocal

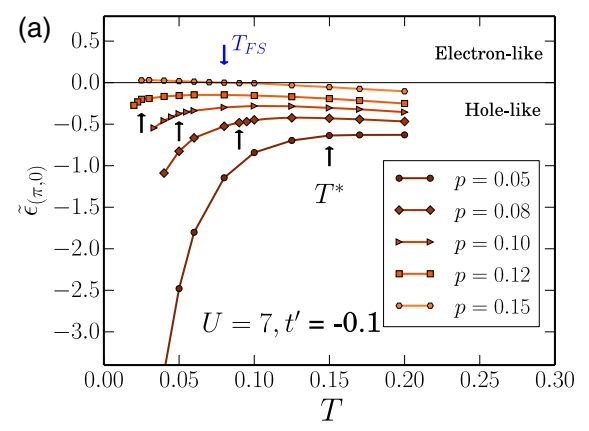

(b)

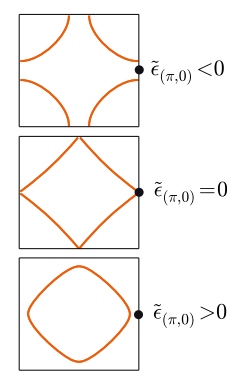

FIG. 3. Antinodal quasiparticle dispersion and Fermi-surface topology. (a) Antinodal quasiparticle energy $\tilde{\epsilon}_{(\pi, 0)}$ for different doping levels, as a function of temperature. The pseudogap onset temperature $T^{*}$ and the Lifshitz transition temperature $T_{\mathrm{FS}}$ are indicated by arrows. Below the pseudogap temperature, $\tilde{\epsilon}_{(\pi, 0)}$ rapidly becomes very negative, driving the Fermi surface holelike. Only when no pseudogap is present (here, for $p>0.12$ ) does $\tilde{\epsilon}_{(\pi, 0)}$ increase at low temperature, and it eventually becomes positive to yield an electronlike Fermi surface. (b) Illustration of the relation between the sign of $\tilde{\epsilon}_{(\pi, 0)}$ and the Fermi-surface topology. electronic correlations. For this value of $t^{\prime}=-0.1$, the connection between the disappearance of the pseudogap and the recovery of an electronlike surface is clear. Indeed, when no pseudogap is present as, e.g., for $p=0.15, \tilde{\epsilon}_{(\pi, 0)}$ keeps on increasing and crosses zero, and an electronlike Fermi surface is recovered at low $T$.

\section{Particle-hole asymmetry and polelike structure in the self-energy}

From the definition of $\tilde{\epsilon}_{(\pi, 0)}$, it is clear that it is the real part of the self-energy at the antinode that drives the renormalization of the Fermi surface. In Fig. 4(a), we consider a fixed doping level $p=5 \%$ and display $\operatorname{Re} \Sigma_{(\pi, 0)}^{(2)}(\omega=0)$ as a function of $t^{\prime}$, in which $\Sigma^{(2)} \equiv \Sigma-$ $U p / 2$ is the self-energy from which the Hartree (infinite frequency) contribution has been subtracted out. It is seen that $\operatorname{Re} \Sigma_{(\pi, 0)}^{(2)}(\omega=0)$ changes sign around $t^{\prime} \simeq-0.2$ and becomes negative and fairly large for larger values of $t^{\prime}$. This pushes the Fermi-surface topology transition to higher values of $t^{\prime}$ : For 5\% doping, it remains holelike up to $t^{\prime} \simeq+0.2$, whereas the Lifshitz transition of the noninteracting system occurs at $t^{\prime} \simeq-0.05$ (see also Fig. 2).

The real part of the self-energy is related to its imaginary part through the Kramers-Kronig relation,

$\operatorname{Re} \Sigma_{\mathbf{k}}^{(2)}(\omega=0)=\frac{1}{\pi} \int_{0^{+}}^{\infty} \frac{\operatorname{Im} \Sigma_{\mathbf{k}}\left(\omega^{\prime}\right)-\operatorname{Im} \Sigma_{\mathbf{k}}\left(-\omega^{\prime}\right)}{\omega^{\prime}} d \omega^{\prime}$.

It is, therefore, instructive to analyze the behavior of $\operatorname{Im} \Sigma_{(\pi, 0)}(\omega)$ [Fig. 4(b)] for several values of $t^{\prime}$ (as indicated by the points A, B, C, and D in Fig. 2), corresponding to positive, vanishing, and negative values of $\operatorname{Re} \Sigma_{(\pi, 0)}^{(2)}(\omega=0)$. In all four cases, the imaginary part of the self-energy displays a prominent peak, corresponding to a polelike feature of the self-energy. For $t^{\prime}=-0.2$ (point B), this peak is centered at $\omega=0$. Because it is particlehole symmetric, it leads to a vanishing real part of the selfenergy [see Fig. 4(a)]. For values of $t^{\prime}$ just below and above -0.2 (points $A$ and $C$ ), the peak in $\operatorname{Im} \Sigma_{(\pi, 0)}(\omega)$ shifts to negative (positive) values of $\omega$. It has become particle-hole asymmetric and induces a positive (negative) real part of the self-energy. There is, therefore, a direct connection between the existence of a large particle-hole asymmetric peak in the imaginary part of the self-energy and the renormalization of the Fermi surface to a more holelike topology. Note that the largest value of the low-frequency scattering rate as $t^{\prime}$ is varied is found when $\operatorname{Im} \Sigma_{(\pi, 0)}(\omega)$ is particle-hole symmetric (e.g., point B in Fig. 4): This defines the location of the red line in Fig. 2 (see also the Appendix B). Anywhere above this line, the self-energy is particle-hole asymmetric and drives the Fermi-surface topology transition to larger doping $p$, as compared to the noninteracting case. Note that the system becomes very 

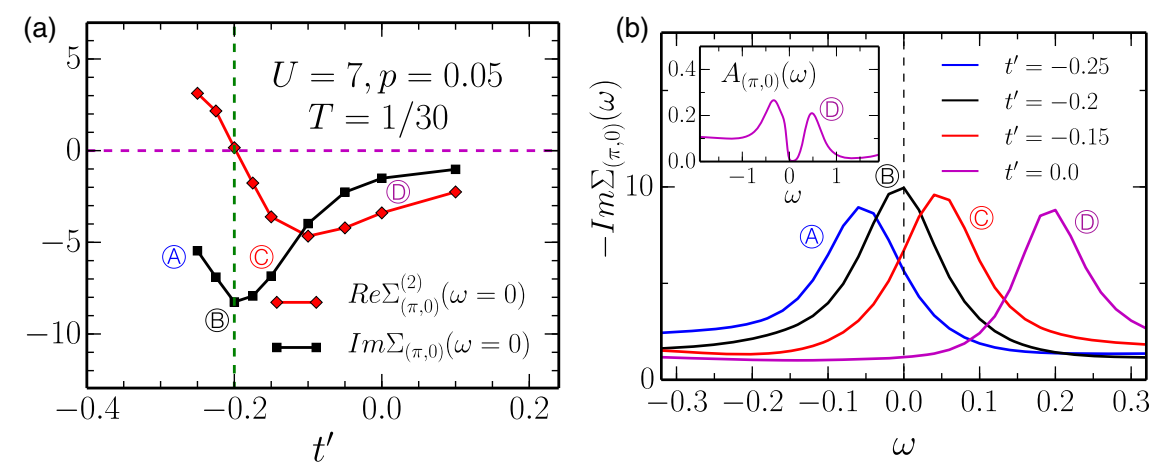

FIG. 4. Evolution of the antinodal self-energy at fixed doping $p=0.05$, as a function of $t^{\prime}$. (a) Real (Hartree subtracted; see text) and imaginary parts of the antinodal self-energy at $\omega=0$. The real part vanishes where the imaginary part is maximum, corresponding to a particle-hole symmetric low-energy $\operatorname{Im} \Sigma_{(\pi, 0)}(\omega)$. (b) Real-frequency scattering rate $\operatorname{Im} \Sigma_{(\pi, 0)}(\omega)$ obtained from the maximum entropy method for different values of $t^{\prime}$. It displays a polelike feature that crosses zero at $t^{\prime} \simeq-0.2$ (point B) where the low-energy scattering is maximum. When the pole is on the positive energy side, it induces a negative real part of the self-energy (through the Kramers-Kronig relation) that drives the Fermi surface more holelike. Inset: Antinodal spectral function at point $\mathrm{D}$ at $T=1 / 30$. See Fig. 2 for the locations of points $\mathrm{A}-\mathrm{D}$ in the $\left(p-t^{\prime}\right)$ plane.

incoherent below the red line, at more negative values of $t^{\prime}$ and small doping. The precise nature of the Fermi surface in this region, and its possible reconstruction, is difficult to assess with the methods employed here.

This polelike feature in the self-energy is also responsible for opening the pseudogap, as clearly seen from the inset of Fig. 4(b), which displays the antinodal spectral function: The minimum of the spectral intensity is found to coincide with the frequency of the quasipole, where $\operatorname{Im} \Sigma_{(\pi, 0)}(\omega)$ is largest.

\section{Fermi-surface topology: Numerically exact DQMC results}

These results have been obtained using the DCA approximation with an eight-site cluster (see Appendix A). We also cross-checked these results with a different and independent method: numerically exact DQMC [40] at $T=1 / 3$. The result is displayed in Fig. 5 (left panel) and clearly shows that the antinodal self-energy drives the Fermi surface holelike over a broad region of the $\left(p, t^{\prime}\right)$ plane, in agreement with our DCA calculations. One can again observe a line where $\operatorname{Re} \Sigma_{(\pi, 0)}^{(2)}(\omega)$ vanishes, mapped out for several values of $U$ in the right panel. This line compares with the red line of Fig. 2. It moves closer to half-filling as $U$ is increased (see also Fig. 11) and towards the noninteracting Lifshitz transition line as $U$ is reduced.

\section{E. SU(2) gauge theory}

Recent numerical work, using a "fluctuation diagnostics" analysis of the contributions to the electronic selfenergy in both the DCA [26] and lattice diagrammatic Monte Carlo [28] approaches have established that the pseudogap is associated with the onset of short-range antiferromagnetic (AF) correlations. On the analytical side, an $\mathrm{SU}(2)$ gauge theory approach has been introduced [37-39] to deal with states in which AF long-range order is destroyed by orientational fluctuations of the order
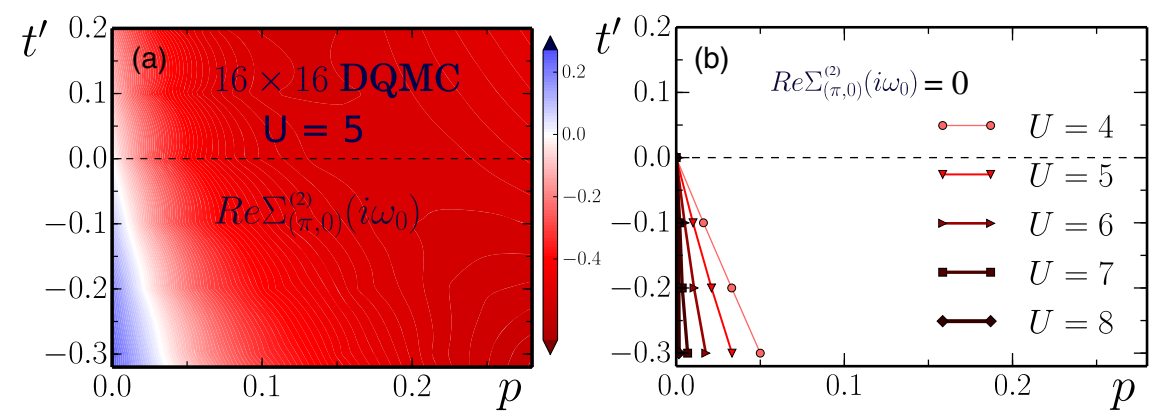

FIG. 5. Particle-hole asymmetry from determinant quantum Monte Carlo (see Appendix A). (a) Real part of the self-energy in the $p$ - $t^{\prime}$ plane for $U=5$. For a broad region (indicated in red), $\operatorname{Re} \Sigma_{(\pi, 0)}^{(2)}$ is negative, hence driving the Fermi surface more holelike, in agreement with our DCA results. (b) The line where $\operatorname{Re} \Sigma_{(\pi, 0)}^{(2)}=0$ and where the antinodal scattering rate is largest is indicated for different values of $U$ (to be compared to the red line in Fig. 2). As $U$ is increased, the region where the Fermi surface is driven holelike becomes larger. 
parameter. It is, thus, very natural to attempt to interpret our numerical results in this framework and compare them to a mean-field treatment of this gauge theory.

This approach is based on the following representation of the physical electron fields on each lattice site $i$ :

$$
\left(\begin{array}{c}
c_{i \uparrow} \\
c_{i \downarrow}
\end{array}\right)=R_{i}\left(\begin{array}{c}
\psi_{i+} \\
\psi_{i-}
\end{array}\right) .
$$

In this expression, $\psi_{ \pm}$are "chargons," fermions that carry charge but no spin quantum numbers, and $R_{i}$ 's are $2 \times 2$ unitary matrix fields, the bosonic spinons $\left(R_{i} R_{i}^{\dagger}=R_{i}^{\dagger} R_{i}=1\right)$. The $R_{i}$ matrix can be thought of as defining the local reference frame associated with the local $\mathrm{AF}$ order (for early work promoting the local reference frame to a dynamical variable, see Refs. [45-47]). This representation has a local gauge invariance corresponding to $R_{i} \rightarrow R_{i} V_{i}^{\dagger}, \psi_{i} \rightarrow V_{i} \psi_{i}$, with $V_{i}$ an $\mathrm{SU}(2)$ matrix. The Hubbard interaction can be decoupled using a vector field $\Phi_{i}$ conjugate to the local spin-density $c_{i \alpha}^{\dagger} \sigma_{\alpha \beta} c_{i \beta} / 2$, and a vector "Higgs field" is introduced such that

$$
\boldsymbol{\sigma} \cdot \boldsymbol{H}_{i}=R_{i}^{\dagger} \boldsymbol{\sigma} R_{i} \cdot \boldsymbol{\Phi}_{i} .
$$

This identifies the Higgs field $\boldsymbol{H}_{i}$ as the local antiferromagnetic moment in the rotated reference frame. Note that $\boldsymbol{H}_{i}$, which transforms under the adjoint of the gauge $\mathrm{SU}(2)$, does not carry any spin, since it is invariant under a global spin rotation.

We can now consider Higgs phases in which $\left\langle\boldsymbol{H}_{i}\right\rangle \neq 0$ but $\left\langle R_{i}\right\rangle=0$. Because of the latter, such phases do not display long-range $\mathrm{AF}$ order, which has been destroyed by orientational fluctuations. However, $\left\langle\boldsymbol{H}_{i}\right\rangle \neq 0$ signals that the local order has a nonzero amplitude. A nonzero $\left\langle\boldsymbol{H}_{i}\right\rangle$ also implies that such a phase has topological order, corresponding to different possible residual gauge groups once the SU(2) gauge symmetry has been spontaneously broken by the Higgs condensate [48-51]. There are different possible mean-field solutions for the Higgs condensate, corresponding to different topological orders and different broken discrete symmetries [38]. Here, we shall focus on the simplest one with $\mathrm{U}(1)$ topological order that preserves all space group, time-reversal, and spin rotations symmetries; this corresponds to the following configuration of the Higgs field (which resembles AF order):

$$
\left\langle\boldsymbol{H}_{i}\right\rangle=\left(0,0, H_{0} e^{i \boldsymbol{Q} \cdot \boldsymbol{R}_{i}}\right),
$$

in which $H_{0}$ is the Higgs field amplitude and $\boldsymbol{Q}=(\pi, \pi)$.

Solving the gauge theory at the mean-field level, the Green's function and self-energy of the chargon field is easily calculated. Because the chargon field "sees" an antiferromagnetic environment, it is identical to the expression obtained for an antiferromagnetic spin-density wave [39]. It thus has a matrix form that involves both components that are diagonal in momentum and offdiagonal components coupling $\boldsymbol{k}$ to $\boldsymbol{k}+\boldsymbol{Q}$ :

$$
G_{\psi}(\omega, \boldsymbol{k})^{-1}=\left(\begin{array}{cc}
\omega-\xi_{\boldsymbol{k}}^{\psi} & H_{0} \\
H_{0} & \omega-\xi_{\boldsymbol{k}+\boldsymbol{Q}}^{\psi}
\end{array}\right) .
$$

Its momentum diagonal component reads

$$
\begin{aligned}
G_{\psi}(\omega, \boldsymbol{k}) & =\left[\omega-\xi_{\boldsymbol{k}}^{\psi}-\Sigma_{\psi}(\omega, \boldsymbol{k})\right]^{-1} \\
\Sigma_{\psi}(\omega, \boldsymbol{k}) & =\frac{H_{0}^{2}}{\omega-\xi_{\boldsymbol{k}+\boldsymbol{Q}}^{\psi}+i 0^{+}} .
\end{aligned}
$$

In this expression, $\xi_{k}^{\psi}=-2 Z_{t} t\left(\cos k_{x}+\cos k_{y}\right)-$ $4 Z_{t^{\prime}} t^{\prime} \cos k_{x} \cos k_{y}-\mu$ is the renormalized dispersion of the chargons. A quantitative calculation of the renormalization factors $Z_{t}$ and $Z_{t^{\prime}}$ requires a full solution of the mean-field equations. We found typical values $Z_{t} \sim 0.3$ and $Z_{t^{\prime}} \sim 0.2$, weakly dependent on the doping level $p$, since the chemical potential mainly affects the chargon dispersion but not the spinon dispersion. Importantly, the self-energy [Eq. (8)] of the chargons has a pole at $\omega_{k}=\xi_{k+Q}^{\psi}$. Hence, the mean-field chargon Green's function has zeros: These zeros are located at zero energy on the Brillouin zone contour defined by $\xi_{\boldsymbol{k}+\boldsymbol{Q}}^{\psi}=0$, corresponding to a chargon "Luttinger surface." There are two bands of chargon excitations, corresponding to the solutions of $\left(\omega-\xi_{k}^{\psi}\right)\left(\omega-\xi_{k+Q}^{\psi}\right)-H_{0}^{2}=0$. To summarize, a crucial aspect of this SU(2) gauge theory description is to have chargons whose dispersions are identical (at the mean-field level) to the excitations of a spin-density wave states, despite the theory having no long-range order or broken symmetries (i.e., the symmetry is restored by the fluctuations of the spinon fields).

At the mean-field level, in the phase associated with the configuration of the Higgs field considered here, the spinon excitations are gapped. In order to obtain the physical electron Green's function, a convolution of the chargon and spinon Green's function over frequency and momentum must be performed, $G_{c}=G_{R} \star G_{\psi}$, and the physical electron self-energy can then be obtained from $\Sigma=\omega+\mu-$ $\epsilon_{k}-G_{c}^{-1}$ (with $\epsilon_{k}$ the bare dispersion defined above). For the purpose of the present paper, a detailed discussion of the spinon dispersion and Green's function is not essential; see Appendix F and Ref. [39] for details. It is sufficient here to emphasize the two following points. (i) The convolution mainly broadens the pole structure of $G_{\psi}$, but the locations in momentum and frequency of the most singular structures of the physical self-energy are still those encoded in the chargon self-energy given by Eq. (8). (ii) The convolution does bring an important effect, however: In contrast to the imaginary part of the chargon self-energy, which is constant all along the Luttinger surface $\xi_{\mathbf{k}+\mathbf{Q}}^{\psi}=0$, the imaginary part of the physical electron self-energy obtained from the 
convolution of Green's functions has an imaginary part that is larger close to the antinodes than close to the nodes; see Fig. 15 in Appendix F. Hence, the gauge theory manages to capture qualitative aspects of the nodalantinodal dichotomy found in our DCA calculations.

The figure also shows that the peak frequency $\omega_{p}$ shifts from negative to positive frequency as $t^{\prime}$ is increased. The inset of this figure displays the corresponding spectral function at the antinode, which has a pseudogap caused by the quasipole at $\omega_{p}$. Note that the pseudogap is particlehole asymmetric, as expected from the fact that it does not originate from the particle-particle channel. These results are in excellent qualitative agreement with the DCA calculations above (Fig. 4). Note that, for the sake of comparison to the finite-temperature DCA results, the gauge theory calculations presented here are performed at a finite temperature larger than the spinon gap. How gapless nodal excitations survive in the gauge theory description as temperature is lowered below this gap (e.g., by having bound states of the chargons and spinon as in a Fractionalized Fermi liquid (FL*) state [52]) is an important question, which is, however, beyond the scope of the present paper.

In Fig. 6(a), we summarize important aspects of the mean-field analysis of the gauge theory [39] as a function of doping level $p$ and $t^{\prime}$. As in Fig. 2, the blue line in this figure is the location of the Lifshitz transition of the physical electron Fermi surface from holelike to electronlike [as defined by the change of sign of the renormalized antinodal dispersion, Eq. (2)], and the red line indicates where $\omega_{p}=0$ (i.e., where particle-hole symmetry is approximately restored at low energy). In good qualitative agreement with the DCA results displayed in Fig. 2, one sees that the Lifshitz transition of the physical FS is pushed to much larger doping in comparison to that of the noninteracting system (dashed line), and that the location of the red line where the pole is close to zero energy is pushed to much smaller doping. The latter approximately coincides with the Lifshitz transition of the chargon Luttinger surface, given by $4 Z_{t^{\prime}} t^{\prime}=\mu$. Because the chemical potential $\mu$ of the interacting system takes more negative values than the noninteracting one and also because $Z_{t^{\prime}}<1$, the red line is shifted to lower doping as $U$ increases, in agreement with the result of Fig. 5. This clarifies why the pole is found at positive energies for most values of $\left(p-t^{\prime}\right)$ and why the Fermi surface is driven holelike in a wide region of the $\left(p-t^{\prime}\right)$ plane. A striking consequence of the presence of the pole is illustrated around the $t^{\prime}=0, p=0$ point, corresponding to the half-filled Hubbard model with only nearest-neighbor hopping, in which the antinodal scattering must be particle-hole symmetric by symmetry. When the system is very slightly hole-doped away from $p=0$, both DCA and the mean-field gauge theory suggest that the particle-hole symmetric point rapidly shifts to very negative $t^{\prime}$. This is in striking contrast to weak-coupling theories in which approximate particle-hole symmetry at the antinode would be restored at the noninteracting Lifshitz transition (dashed line). We note that there are quantitative discrepancies in the location of these two lines between the numerical DCA results and the mean-field gauge theory results, which are predominantly due to the assumptions made on the renormalization parameters $Z_{t}$ and $Z_{t^{\prime}}$ entering the chargon dispersion and on the Higgs field amplitude $H_{0}$.

Importantly, the mean-field analysis of the SU(2) gauge theory provides a physical understanding of the origin of the pseudogap and of the quasipole of the self-energy [see Fig. 6(b)] as being due to short-range antiferromagnetic correlations, long-range order being destroyed by orientational fluctuations. The quasipole is responsible for the pseudogap in the physical electron Green's function, while the spinon (R) spectrum displays a gap. The chargons have
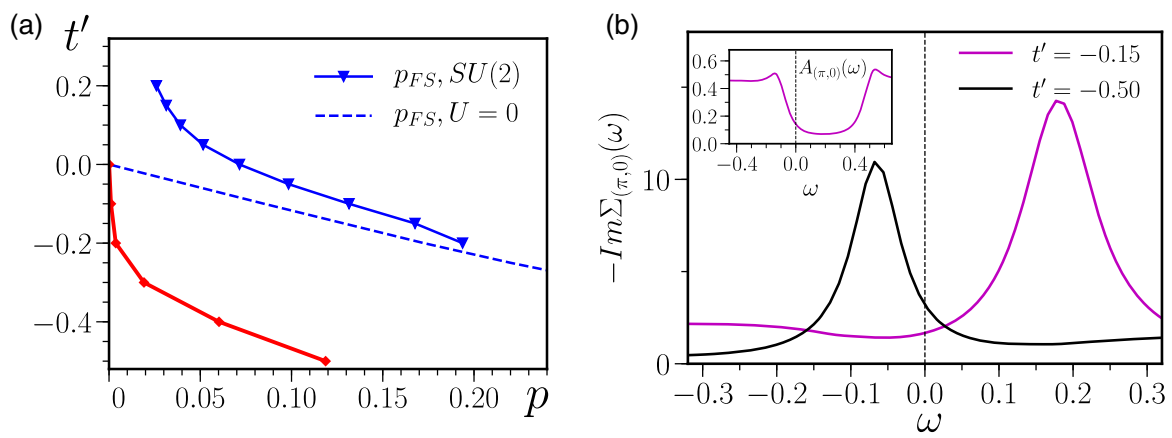

FIG. 6. Pseudogap and FS topology within the SU(2) gauge theory at the mean-field level. Color coding is identical to Fig. 2 (DCA results), to which this figure should be compared. (a) Solid blue line: Lifshitz transition of the interacting Fermi surface. Along the red line, the self-energy pole is at zero energy and approximate particle-hole symmetry is restored. This also corresponds to the Lifshitz transition of the chargons. (b) Electronic self-energy at the antinode for two different values of $t^{\prime}$. The quasipole in the self-energy moves from negative to positive frequency as $t^{\prime}$ is increased; see Fig. 4. The inset displays the antinodal spectral function for $t^{\prime}=-0.15$, emphasizing that its minimum coincides with the position of the pole. Here we assumed $H_{0}=0.3$, a spinon gap $\Delta=0.01$, and $J=0.1$ (the nearest-neighbor coupling of the spin-wave fluctuations; see Ref. [39]). A broadening factor $\eta=0.04$ is used to obtain smooth spectral functions. 
a spectrum characteristic of an $\mathrm{AF}$ spin-density wave despite the absence of AF long-range order, and their self-energy has a sharp pole at the mean-field level. The (red) line where the pole crosses zero energy, corresponding to an approximate restoration of particle-hole symmetry at low energy, can be interpreted [39] as the Lifshitz transition of the chargon Luttinger surface.

\section{DISCUSSION AND EXPERIMENTAL RELEVANCE}

Our results establish that an asymmetric polelike feature in the antinodal self-energy is responsible for both the pseudogap and for the renormalization and topological transition of the FS. We note that, in weak-coupling approaches such as spin-fluctuation theories (see Appendix $\mathrm{E}$ for a detailed discussion), the self-energy becomes very large for $\omega=\epsilon_{\mathbf{k}+(\pi, \pi)}-\mu$, provided that the antiferromagnetic correlation length $\xi$ is large enough and that $v_{F} / \xi<T$. As a result, hot spots form on the Fermi surface, at specific k-vectors defined by $\epsilon_{\mathbf{k}}=\epsilon_{\mathbf{k}+(\pi, \pi)}=\mu$, corresponding to the intersection of the antiferromagnetic Brillouin zone with the Fermi surface. Hence, in a weakcoupling approach, the change of sign of the bare dispersion $\epsilon_{(\pi, 0)}-\mu=0$ controls both the doping at which the hot spots reach the antinode and that where the Lifshitz transition occurs. As a result, the noninteracting Fermi surface transition line (blue dashed line in Fig. 2) controls at the same time the location of the Lifshitz transition, the symmetry of the self-energy, and the suppression of spectral weight along the Fermi surface. This is in stark contrast to our strong-coupling results, where these phenomena appear at distinct locations. In particular, we have demonstrated that the line in $\left(p-t^{\prime}\right)$ parameter space where particle-hole symmetry is approximately obeyed at low energy is pushed, at strong coupling, to very low values of $p$ and very negative values of $t^{\prime}$; see Figs. 2 and 5 where this line is displayed in red. This is crucial in explaining why interactions drive the Fermi surface more holelike for a wide range of $\left(p-t^{\prime}\right)$, where the noninteracting (or weak coupling) FS would actually be electronlike, and why the Lifshitz transition is pushed to larger values of $p$ in comparison to the noninteracting system.

In order to put our results into perspective, we note that the relation between a polelike feature in the self-energy and the pseudogap, as well as the implications of the corresponding zeros of the Green's function for the reconstruction of the Fermi surface, has been previously discussed in cluster extensions of dynamical mean-field theory $[22,23,30,32,34-36]$ and in phenomenological theories such as Yang-Rice-Zhang (YRZ) theory [53] or other approaches [54,55] (see Ref. [56] for a gauge-theory perspective on the YRZ phenomenology). The existence of a Lifshitz transition as the hole doping is increased was also discussed in some previous cluster DMFT or DCA studies [30-32]. However, the role played by the particle-hole asymmetry associated with the self-energy pole in determining the FS topology and the systematic dependence of this asymmetry on $\left(p-t^{\prime}\right)$ were not unraveled and studied and, hence, the key interplay between FS topology and the pseudogap was not previously revealed.

We now discuss the relevance of our results to experiments on hole-doped cuprates. We first note that, indeed, a pseudogap is not found when the Fermi surface is electronlike and, hence, that the relation $p^{*} \leq p_{\mathrm{FS}}$ is apparently obeyed in all compounds. In the single-layer compound $\mathrm{La}_{2-x} \mathrm{Sr}_{x} \mathrm{CuO}_{4}$ (LSCO), with a small value [57] of $\left|t^{\prime} / t\right|$, the in-plane resistivity in high magnetic fields [58] suggests that $p^{*} \simeq 0.18$. Currently available ARPES experiments [59-61] allow us to ascertain that $0.17<p_{\mathrm{FS}} \lesssim 0.20$. In the Nd-LSCO compound, high-field transport [62] finds $p^{*} \simeq 0.23$, while ARPES [63] has $0.20<p_{\mathrm{FS}}<0.24$.

In another single-layer compound $(\mathrm{Bi}, \mathrm{Pb})_{2}(\mathrm{Sr}, \mathrm{La})_{2} \times$ $\mathrm{CuO}_{6+\delta}(\mathrm{Bi2201})$ [64-67], it is found that $p^{*} \simeq p_{\mathrm{FS}}$. An ARPES experiment on the bilayer Bi2212 material [68] has shown that the antibonding Fermi surface crosses the antinode at $p_{\mathrm{FS}} \simeq 0.22$ and suggested that it may be connected to the onset of the pseudogap. This was further confirmed in a recent electronic Raman experiment $[3,4]$ that found the pseudogap end point at $p^{*} \simeq 0.22$. Note that the Raman response is believed to be predominantly sensitive to the antibonding band since it is close to a density of states singularity [3] and does not give information about the possible existence of a pseudogap in the bonding band (which remains holelike for all dopings). In compounds with larger values [57] of $\left|t^{\prime} / t\right|$, such as $\mathrm{YBa}_{2} \mathrm{Cu}_{3} \mathrm{O}_{7-\delta} \quad[69,70], \quad \mathrm{Tl}_{2} \mathrm{Ba}_{2} \mathrm{CuO}_{6+\delta} \quad[71,72]$, or $\mathrm{HgBa}_{2} \mathrm{CuO}_{4+\delta}$ [73], it is generally believed that $p_{\mathrm{FS}}$ and $p^{*}$ are distinct with $p^{*}<p_{\mathrm{FS}}$. This is in qualitative agreement with our finding that the FS and pseudogap critical doping coincide for smaller values of $\left|t^{\prime} / t\right|$ and are distinct for larger ones. Hence, we conclude on the basis of our results and experimental observations that there are two families of hole-doped cuprates: materials with smaller values of $\left|t^{\prime} / t\right|$, for which the collapse of the pseudogap and change of FS topology coincide $\left(p^{*} \simeq p_{\mathrm{FS}}\right)$, and materials with larger values of $\left|t^{\prime} / t\right|$, for which these are distinct phenomena $\left(p^{*}<p_{\mathrm{FS}}\right)$.

Finally, a very recent study on Nd-LSCO using hydrostatic pressure to tune the band structure found that both $p_{\mathrm{FS}}$ and $p^{*}$ decrease by the same amount [5]. This provides a compelling experimental demonstration that $p^{*}$ cannot exceed $p_{\text {FS }}$.

We finally comment on the predicted renormalization of the Fermi surface by strong correlations. In view of Fig. 2, the materials for which this effect is expected to be strongest are the ones with smaller values of $\left|t^{\prime} / t\right|$; hence, we turn to LSCO. We note that, in order to fit the ARPES Fermi surface using a single-band tight binding model, the effective parameter $t^{\prime}$ has to be tuned systematically more negative [corresponding to a more negative $\tilde{\epsilon}_{(\pi, 0)}$ ] as doping 
is reduced, i.e., from $t^{\prime} / t=-0.12$ for $p=0.3$ to $t^{\prime} / t=$ -0.2 for $p=0.03$ [60]. Moreover, electronic structure calculations based on density functional theory-local-density approximations (LDA) yield $p_{\mathrm{FS}} \simeq 0.15$, while ARPES finds $0.17<p_{\mathrm{FS}} \lesssim 0.20$, as mentioned above. These two observations suggest that correlation effects indeed generally drive the Fermi surface more holelike.

\section{CONCLUSION AND OUTLOOK}

To conclude, we have investigated the interplay between the pseudogap and the Fermi-surface topology in the twodimensional Hubbard model. In the weak-coupling regime, these issues are directly connected: hot spots can only form when the Fermi surface is holelike and intersects the antiferromagnetic zone boundary. At stronger coupling, the antiferromagnetic correlations responsible for the pseudogap become short ranged, and it becomes a fundamental puzzle to understand whether there is any connection to FS topology, which is in essence long-distance physics. We provide an answer to this puzzle by showing that a common polelike feature in the electronic self-energy controls both issues. This pole induces a large low-energy scattering rate responsible for the onset of the pseudogap, and its asymmetry leads to significant modifications of the Fermi surface with respect to its noninteracting shape and controls the location of the Lifshitz transition. As a consequence, we find that the pseudogap only appears on holelike Fermi surfaces, i.e., $p^{*} \leq p_{\mathrm{FS}}$, and that $p^{*} \simeq p_{\mathrm{FS}}$ for an extended range of doping levels and values of $t^{\prime}$. These findings are in good agreement with available experimental data. We have also shown that our results can be interpreted in the framework of an SU(2) gauge theory of fluctuating antiferromagnetism with topological order. This provides an explanation for the origin of the pole in the self-energy and establishes the connection between the pseudogap and the Fermi-surface topology through the chargon Luttinger surface. This effort to bridge the gap between numerical results obtained within cluster extensions of DMFT and low-energy effective field theories is pursued and detailed in a companion publication [39].

Let us emphasize that in most of the parameter range relevant to hole-doped cuprates, the self-energy pole is found at a positive energy. Hence, the strongest suppression of the antinodal spectral weight is predicted to occur at energies above the Fermi level, which is not directly accessible to ARPES experiments. While a strong particle-hole asymmetry is indeed observed by scanning tunneling microscopy (STM) [74,75], this emphasizes again [76] the importance of developing momentumresolved spectroscopies able to probe the "dark side" of the Fermi surface. Finally, an outstanding question is to explore whether the topological order, associated with the pseudogap regime in the gauge theory description, can be revealed more directly in numerical studies of Hubbard-like models.

\section{ACKNOWLEDGMENTS}

We are grateful to L. Taillefer and N. Doiron-Leyraud for sharing and discussing their experimental data before publication. We also acknowledge discussions with $\mathrm{M}$. Civelli, M. Kim, G. Kotliar, A. J. Millis, O. Parcollet, I. Paul, A. Sacuto, and A.-M. S. Tremblay. This work was supported by the Simons Foundation Many-Electron Collaboration; the European Research Council (Project No. 319286-"QMAC"); the Swiss National Supercomputing Centre (CSCS, Project No. s575); the NSF under Grant No. DMR-1664842; and MURI Grant No. W911NF-14-1-0003 from ARO. The Flatiron Institute is supported by the Simons Foundation (A. G.). Research at the Perimeter Institute (S. S.) is supported by the Government of Canada through Industry Canada and by the Province of Ontario through the Ministry of Research and Innovation. M. S. acknowledges support from the German National Academy of Sciences Leopoldina through Grant No. LPDS 2016-12. S. S. acknowledges support from Cenovus Energy at the Perimeter Institute and from the Hanna Visiting Professor program at Stanford University. A. G. and S. S. are grateful for the stimulating atmosphere of the Jouvence workshop.

\section{APPENDIX A: METHODS}

Our results for the two-dimensional Hubbard model are obtained using two methods: unbiased DQMC [40] and the DCA [15,77], a cluster extension of DMFT [78] that captures the physics of short-range spatial correlations. We perform DQMC on a $16 \times 16$ lattice with periodic boundary conditions at a temperature $T=1 / 3$. Since the inverse temperature $\beta=T^{-1}=3$ is significantly smaller than the linear size of the lattice $L=16$, the finite size effects are negligible in the DQMC calculation. The imaginary time step was set to $\Delta \tau=3 / 64$, which is small enough to avoid artifacts due to the discretization errors. We use $5.12 \times 10^{5}$ Monte Carlo sweeps to collect the data after 1000 warmup sweeps.

The DCA calculation is performed with an eight-site cluster. In the DCA approach, the lattice self-energy is approximated by a patchwise-constant self-energy $\Sigma_{\mathbf{K}}$ in the Brillouin zone. We solved the DCA equations with an eight-site auxiliary quantum impurity cluster. In the geometry we used, the Brillouin zone is divided into eight sectors where the self-energy is constant, as shown in Fig. 7. Note that there are clearly distinct patches for the antinodal and the nodal regions of the Brillouin zone.

We use both the Hirsch-Fye [77] and the continuoustime quantum Monte Carlo [79] method to solve the auxiliary cluster impurity problem. A comparison of both methods shows that the imaginary-time step $\Delta \tau=1 / 21$ used in the Hirsch-Fye solver is small enough, as shown in Appendix B. We use 50 DMFT iterations to get a converged result and use $(2-10) \times 10^{6}$ Monte Carlo sweeps at each 


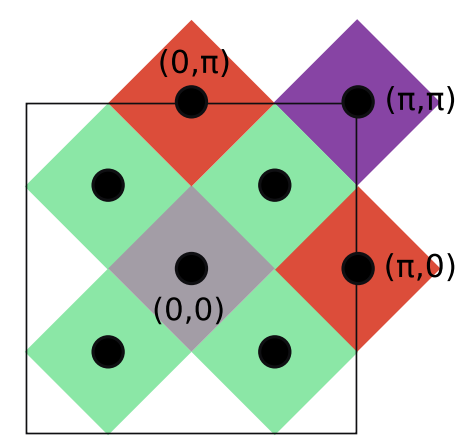

FIG. 7. The patches in momentum space of the eight-site DCA method. The self-energy is constant over each patch.

iteration. In order to have better statistics, the results are averaged over the last few converged iterations. The typical statistical error in the real part of the self-energy and the spectral intensity at zero frequency is approximately $1 \%$.

We identify the pseudogap temperature $T^{*}$ as the maximum of the temperature dependent spectral weight $A_{(\pi, 0)}(\omega=0) \equiv-(1 / \pi) \operatorname{Im} G_{(\pi, 0)}(\omega=0)$. It is obtained from a linear extrapolation to zero frequency of $-\operatorname{Im} G_{(\pi, 0)}\left(i \omega_{n}\right)$ at the first two Matsubara frequencies. We find $T_{\mathrm{FS}}$ from the zero of the effective dispersion at the antinode $\tilde{\epsilon}_{(\pi, 0)}$.

Finally, the real-frequency spectral function $A_{\mathbf{k}}(\omega)$ and the self-energy $\Sigma_{\mathbf{k}}(\omega)$ are found with the maximum entropy analysis $[80,81]$ on the Green's function $G_{\mathbf{k}}\left(i \omega_{n}\right)$ and the self-energy $\Sigma_{\mathbf{k}}\left(i \omega_{n}\right)$. We use two independent maximum entropy codes $[80,81]$ to make sure that their results agree.

\section{APPENDIX B: SUPPORTING MATERIAL FOR THE RESULTS SHOWN IN THE MAIN TEXT}

\section{Pseudogap onset temperature $T^{*}$ and Fermi-surface topology transition temperature $T_{\mathrm{FS}}$}

We identify the pseudogap onset temperature $T^{*}$ as the temperature where the spectral function at the antinodal point $A_{(\pi, 0)}(\omega=0)$ reaches a maximum as temperature is lowered; see Fig. 8. The zero-frequency value of the spectral function $A_{(\pi, 0)}(\omega=0)=-(1 / \pi) \operatorname{Im} G_{(\pi, 0)}(\omega=0)$, which is obtained by a linear extrapolation of the value of $\operatorname{Im} G_{(\pi, 0)}\left(i \omega_{n}\right)$ (the result of the numerical calculation) at its first two Matsubara frequencies. We found that different approximations of the spectral function, such as $A_{(\pi, 0)}(\omega=0) \simeq \beta G_{(\pi, 0)}[\tau=(\beta / 2)]$ [82], yield the same values of $T^{*}$. Also, we have used different imaginary time discretization steps $\Delta \tau$ in the Hirsch-Fye algorithm and observe that the results are the same for all values of $\Delta \tau \leq 0.1$. We have used $\Delta \tau=0.0476$ throughout our work.

When the Fermi surface crosses $k=(\pi, 0)$, it undergoes a Lifshitz transition and changes from holelike to electronlike. We define the Fermi surface at finite temperature by the location of the maximum spectral intensity at zero energy (as seen in an ARPES experiment). This maximum goes through $(\pi, 0)$ when the quasiparticle effective
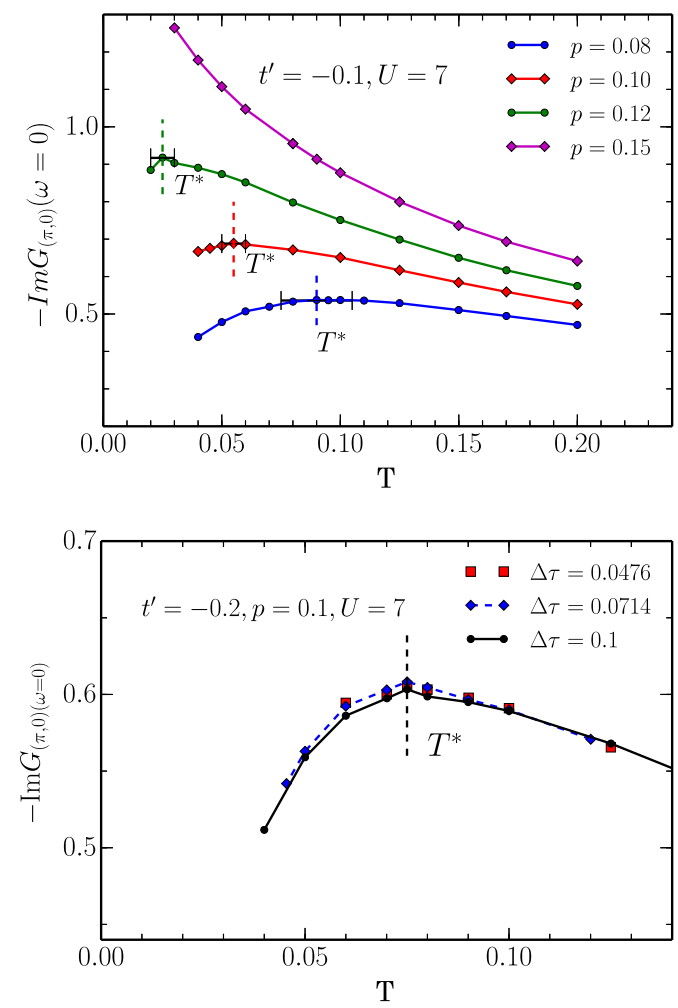

FIG. 8. $-\operatorname{Im} G_{(\pi, 0)}(\omega=0)$ as a function of temperature $T$. The maximum is identified as the pseudogap onset temperature $T^{*}$. Upper panel: For $t^{\prime}=-0.1$ and different doping levels. Lower panel: For $t^{\prime}=-0.2, p=0.1$ and different discrete time steps $\Delta \tau$ of the Hirsch-Fye impurity solver. We can see that $T^{*}$ is already converged for $\Delta \tau=0.1$.

dispersion at the antinode $\tilde{\epsilon}_{(\pi, 0)}=0$, as discussed in the main text [see Eq. (1)]. We can, therefore, find the Fermisurface topology transition temperature $T_{\mathrm{FS}}$ by finding the zero of $\tilde{\epsilon}_{(\pi, 0)}$ as a function of temperature, as shown in Fig. 9. We also display the results as obtained using two

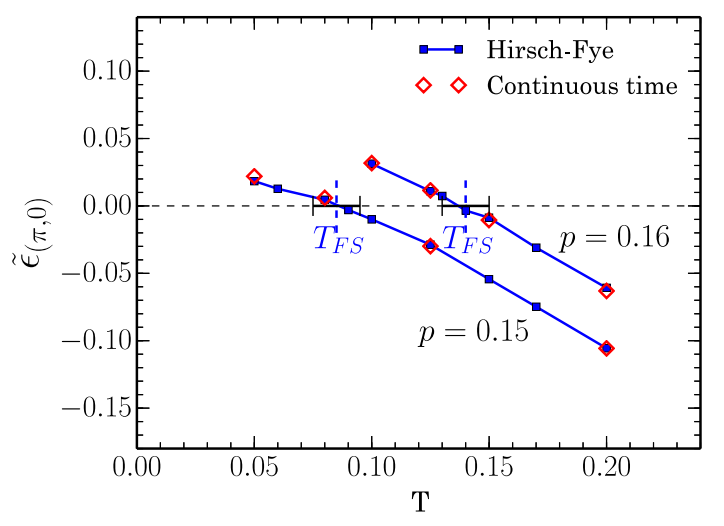

FIG. 9. The effective quasiparticle dispersion at the antinode $\tilde{\epsilon}_{(\pi, 0)}$ as a function of temperature $T$ for two doping levels. At the Fermi-surface topology transition temperature $T_{F S}$, we have $\tilde{\epsilon}_{(\pi, 0)}=0$. We show results using both a continuous-time QMC [79] and a Hirsch-Fye [77] impurity solver (the latter with a finite imaginary-time step $\Delta \tau=0.0476$ ). 

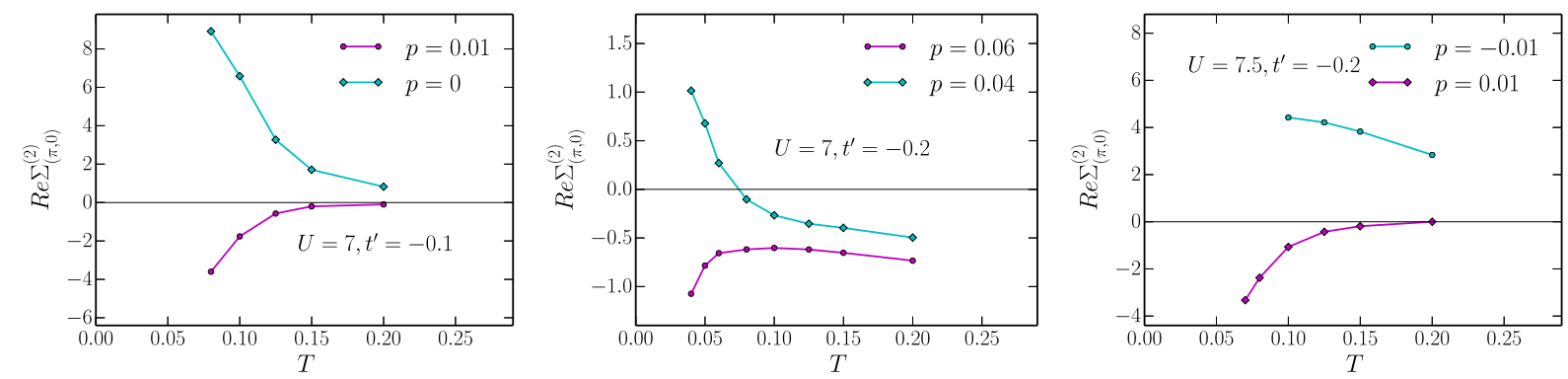

FIG. 10. The real part of the antinodal self-energy as a function of temperature for different dopings $p$ and $t^{\prime}$. Note that the constant Hartree shift has been removed. Left panel: For $U=7, t^{\prime}=-0.1$, at $p=0, \operatorname{Re} \Sigma_{(\pi, 0)}^{(2)}(\omega=0)$ is positive at low temperatures, while at $p=0.01$, it is negative, suggesting that $p_{c}$ is between 0 and 0.01 . Central panel: For $U=7, t^{\prime}=-0.2$, we have $0.04<p_{c}<0.06$. Right panel: For $U=7.5, t^{\prime}=-0.2$, we find $-0.01<p_{c}<0.01$. Therefore, for fixed $t^{\prime}$, increasing $U$ drives $p_{c}$ closer to 0 (half-filling).

different impurity solvers and show that they give identical results.

\section{Maximum low-energy scattering line and particle-hole asymmetry}

As we have shown in the main text, there is a curve in the $p-t^{\prime}$ diagram that separates a region where the polelike feature in the imaginary part of the antinodal self-energy is on the negative energy side from a region where it is on the positive energy side. When the pole is on the positive energy side, the real part of the self-energy (with the Hartree term removed) $\operatorname{Re} \Sigma_{(\pi, 0)}^{(2)}(\omega=0)$ is negative, while it is positive when the pole is on the negative side. We can, therefore, locate the curve by finding, at fixed $t^{\prime}$, the value of the doping $p_{c}$ at which the zero-temperature extrapolation of $\operatorname{Re} \Sigma_{(\pi, 0)}^{(2)}(\omega=0)$ changes sign; see Fig. 10.

\section{3. $U$ dependence of the connection between $p^{*}$ and $p_{\mathrm{FS}}$}

In the main text, all calculations have used $U=7$. For this value, we have shown that $p^{*} \simeq p_{\mathrm{FS}}$ for values of $t^{\prime}$ greater than $\simeq-0.1$. For more negative values of $t^{\prime}$, the $p^{*}$ and $p_{\mathrm{FS}}$ lines split apart. When $U$ is larger, this branching point goes to lower values of $t^{\prime}$. This is shown in Fig. 11,

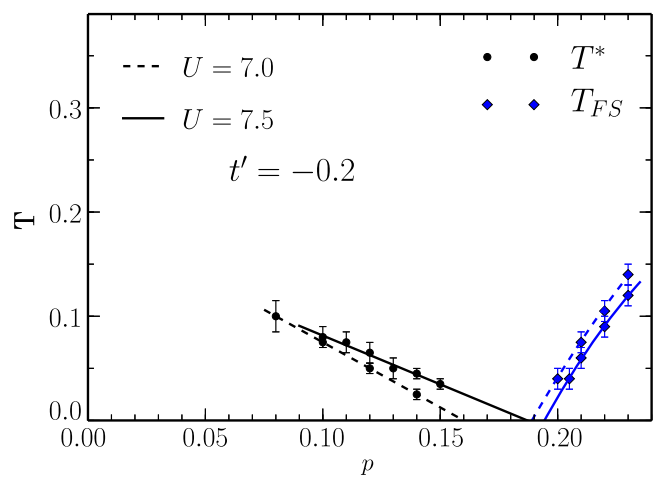

FIG. 11. $T^{*}$ and $T_{\mathrm{FS}}$ as a function of doping $p$ at $t^{\prime}=-0.2$, $U=7$ (dashed lines), and $U=7.5$ (solid lines). Note that here the imaginary-time discretization step is $\Delta \tau=1 / 2 U$. where we compute $T^{*}$ and $T_{\mathrm{FS}}$ for both $U=7$ and $U=7.5$. It is clear from the figure that, for $U=7.5, p^{*}$ and $p_{\mathrm{FS}}$ are much closer than for $U=7$. This can be understood because a larger value of $U$ extends the pseudogap region to larger dopings, while the Fermisurface topology is not influenced much by correlations before we actually have a pseudogap and hence $p^{*} \simeq p_{\mathrm{FS}}$.

\section{APPENDIX C: TEMPERATURE EVOLUTION OF $\tilde{\epsilon}_{k}$ AND ROLE OF NONLOCAL CORRELATIONS}

In Fig. 12, we investigate the role of nonlocal correlations by comparing the results obtained by DCA, as in the main text, and by single-site DMFT that only accounts for local correlations. The black line shows $\tilde{\epsilon}_{(\pi, 0)}$ as computed by DMFT for $t^{\prime}=-0.1$ and $p=0.1$. For these parameters, the noninteracting Fermi surface is electronlike. It is seen that, at low temperature, the DMFT results also predict an electronlike Fermi surface. This is not surprising as DMFT preserves the Luttinger theorem and the interacting Fermi surface is the same as the noninteracting one when $T \rightarrow 0$. However, as temperature is increased, $\tilde{\epsilon}_{(\pi, 0)}$ decreases significantly and becomes negative. This yields a holelike interacting Fermi surface at high temperature that breaks

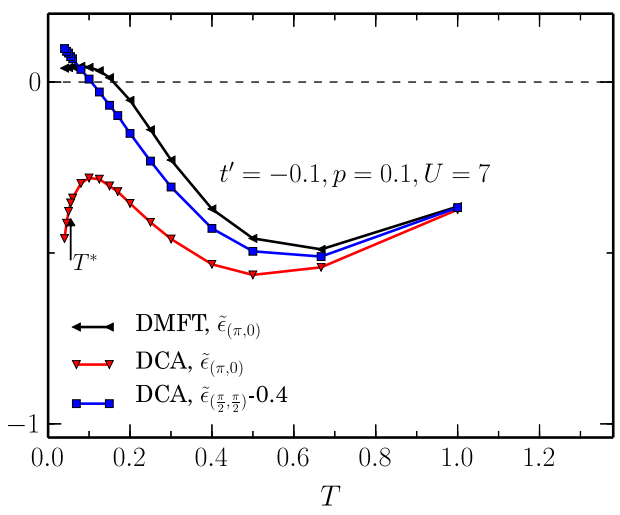

FIG. 12. Temperature dependence of $\tilde{\epsilon}_{k}$ as obtained by DCA and DMFT at $10 \%$ hole doping and $t^{\prime}=-0.1$. 
Luttinger's theorem $[83,84]$ with a volume larger than in the noninteracting case.

The red and blue lines show $\tilde{\epsilon}_{(\pi, 0)}$ and $\tilde{\epsilon}_{[(\pi / 2),(\pi / 2)]}$, respectively, as obtained by DCA. $\tilde{\epsilon}_{[(\pi / 2),(\pi / 2)]}$ has been shifted by a constant $4 t^{\prime}=-0.4$ that corresponds to the energy difference of the noninteracting dispersion at $(\pi, 0)$ and $[(\pi / 2),(\pi / 2)]$. At high temperatures, all curves yield the same value, compatible with a self-energy that is essentially local. As temperature is decreased, the nodal $\tilde{\epsilon}_{[(\pi / 2),(\pi / 2)]}$ behaves like the DMFT solution, indicating that the Fermi surface at the node is very close to its noninteracting shape. The DCA $\tilde{\epsilon}_{(\pi, 0)}$ has a different behavior. As temperature is lowered, it quickly departs from $\tilde{\epsilon}_{[(\pi / 2),(\pi / 2)]}$, showing the onset of nodal-antinodal differentiation. At a temperature slightly above $T^{*}$, nonlocal correlations become large and induce a very negative $\tilde{\epsilon}_{(\pi, 0)}$ as discussed in the main text.

\section{APPENDIX D: PSEUDOGAP AND FERMI-SURFACE TOPOLOGY TRANSITION AS A FUNCTION OF $U$}

Our results for $U=7$ show that, for a broad range of parameters, the pseudogap disappears at the same critical doping where the Fermi surface undergoes a Lifshitz transition. In Fig. 13, we investigate how our results depend on the correlation strength $U$. It is shown that, for values of $U \lesssim 5$, correlations have little effect on the Fermi-surface topology and the low-energy scattering rate $\operatorname{Im} \Sigma_{(\pi, 0)}$ is very small. Above $U \simeq 5$, correlation effects set in quickly, as shown by a fast increase in the value of $\operatorname{Im} \Sigma_{(\pi, 0)}$. This induces a pseudogap at $U=5.6$. At the same time, the effective quasiparticle dispersion $\tilde{\epsilon}_{(\pi, 0)}$ crosses zero and becomes very negative for larger values of $U$. This sudden increase of the correlation effects for $U>5$ might explain

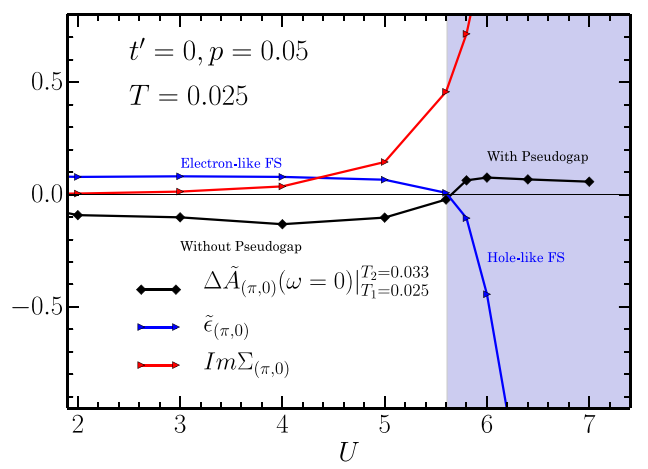

FIG. 13. Correlation effects shown as a function of $U$ for three quantities: the quasiparticle effective dispersion $\tilde{\epsilon}_{(\pi, 0)}$, the imaginary part of the antinodal self-energy at zero energy $\operatorname{Im} \Sigma_{(\pi, 0)}(\omega=0)$, and the difference in spectral intensity at the Fermi level for the two lowest calculated temperatures, indicating whether a pseudogap has formed. why the pseudogap and the Fermi-surface topology happen at the same time.

\section{APPENDIX E: COMPARISON WITH WEAK-COUPLING APPROACHES}

Let us investigate how our results differ from weakcoupling approaches, such as spin-fluctuation theory or the two-particle self-consistent approach (TPSC) of Vilk and Tremblay [85]. The latter has been shown to be quite accurate in the weak to intermediate coupling regime of the two-dimensional Hubbard model, and this appendix closely follows the analysis in Ref. [85].

In those approaches, the self-energy is obtained as

$\Sigma\left(\mathbf{k}, i \omega_{n}\right)=g^{2} T \sum_{p} \frac{1}{V_{\mathrm{BZ}}} \sum_{\mathbf{q}} G_{0}\left(\mathbf{k}+\mathbf{q}, i \omega_{n}+i \nu_{p}\right) \chi\left(\mathbf{q}, i \nu_{p}\right)$,

where $G_{0}$ is the noninteracting Green's function, $\chi$ is the spin susceptibility, and $g$ is a coupling constant with the dimension of energy. When the magnetic correlation length $\xi$ is large, $\chi$ can be approximated by [86]

$$
\chi\left(\mathbf{q}, i \nu_{p}\right) \propto \frac{1}{(\mathbf{q}-\mathbf{Q})^{2}+\xi^{-2} \nu_{p} / \omega_{\mathrm{sp}}+\xi^{-2}},
$$

with $\mathbf{Q}=(\pi, \pi)$ the antiferromagnetic wave vector. The self-energy thus reads

$$
\begin{aligned}
\Sigma\left(\mathbf{k}, i \omega_{n}\right) \propto & T \sum_{p} \frac{1}{V_{\mathrm{BZ}}} \sum_{\mathbf{q}} \frac{1}{i \omega_{n}+i \nu_{p}+\mu-\epsilon_{\mathbf{k}+\mathbf{q}}} \\
& \times \frac{1}{(\mathbf{q}-\mathbf{Q})^{2}+\xi^{-2} \nu_{p} / \omega_{\mathrm{sp}}+\xi^{-2}} .
\end{aligned}
$$

In the regime of interest here (large enough $\xi$, renormalized classical regime), the above sum is dominated by the smallest Matsubara frequency (note that $\omega_{\mathrm{sp}} \sim \xi^{-2}$ ) and one obtains the imaginary part of the retarded realfrequency self-energy in the form

$$
-\frac{1}{\pi} \operatorname{Im} \Sigma_{\text {ret }}(\mathbf{k}, \omega) \propto T \int d^{2} \mathbf{q} \delta\left(\omega-\xi_{\mathbf{k}+\mathbf{q}}\right) \frac{1}{(\mathbf{q}-\mathbf{Q})^{2}+\xi^{-2}} .
$$

The important point is that, in two dimensions, this integral diverges as the correlation length becomes large, which leads to the formation of hot spots at which a pseudogap opens. This integral can actually be performed analytically, and one finally obtains

$$
-\frac{1}{\pi} \operatorname{Im} \Sigma_{\mathrm{ret}}(\mathbf{k}, \omega)=\tilde{g} \frac{T}{\sqrt{\left(\omega-\xi_{\mathbf{k}+\mathbf{Q}}\right)^{2}+\left(v_{F} / \xi\right)^{2}}}+\mathrm{reg},
$$


where "reg." denotes a nonsingular contribution. The physics associated with a weak-coupling description of spin fluctuations can be entirely described on the basis of this expression [85]. Let us focus first on the Fermi surface properties, corresponding to $\omega=0$ and momenta such that $\xi_{\mathbf{k}}=0$. As is clear from Eq. (E5), the self-energy is regular on the Fermi surface except at the hot spots satisfying also $\xi_{\mathbf{k}+\mathbf{Q}}=0$, corresponding to the intersection of the Fermi surface with the antiferromagnetic Brillouin zone. At these hot spots, the self-energy is singular: its imaginary part is of order

$$
-\left.\frac{1}{\pi} \operatorname{Im} \Sigma\right|_{\text {hot }} \propto \frac{T \xi}{v_{F}} .
$$

This is large only when the correlation length is large: $\xi>v_{F} / T$. In this regime, spectral weight is strongly depleted at the hot spots, corresponding to the weakcoupling description of the pseudogap. When the correlation length remains finite as temperature is lowered (short-range order), the hot spots and corresponding pseudogap disappear for $T<v_{F} / \xi$ and conventional Fermi liquid behavior is recovered at low temperature.

Let us emphasize the crucial differences that exist between the weak-coupling expression of the self-energy [Eq. (E5)] and both the self-energy that we obtain from DCA at strong coupling and the self-energy obtained from the SU(2) gauge theory. As is clear from Eq. (E5), the imaginary part of the weak-coupling self-energy does display a peak, but (i) the height of this peak is proportional to $T \xi(T)$ and, thus, eventually the peak and the hot spots disappear at low $T$ if $\xi$ remains finite; (ii) the width of this peak is proportional to $v_{F} / \xi<T$, which in the regime where the peak exists is smaller than temperature. In contrast, in the strong-coupling DCA calculations, the peak is not suppressed as $T$ is reduced, and its width is larger than $T$. Furthermore, the correlation length that we can estimate in our DCA results from the static staggered susceptibility $\chi_{A F} \propto \xi^{2}$ is quite small at strong coupling. We find, for example, $\xi / a \simeq 2.7$ for $U=7, t^{\prime}=-0.2$, $p=0.1$ at $T=1 / 30$. The weak-coupling expression also has a different structure than the singular delta-function form of the chargon self-energy in the SU(2) gauge theory: The latter, importantly, does not involve the correlation length (set by the spinons) and is similar to that of a spin density wave (SDW) in the ordered phase.

The real part of the self-energy corresponding to Eq. (E5) can be obtained using Kramers-Kronig relations as

$$
\operatorname{Re} \Sigma_{\text {ret }}(\mathbf{k}, \omega) \propto \frac{T}{\sqrt{\Omega_{k}^{2}+\left(v_{F} / \xi\right)^{2}}} \ln \left|\frac{\Omega_{k}+\sqrt{\Omega_{k}^{2}+\left(v_{F} / \xi\right)^{2}}}{\Omega_{k}-\sqrt{\Omega_{k}^{2}+\left(v_{F} / \xi\right)^{2}}}\right|,
$$

in which we have used the short-hand notation $\Omega_{k} \equiv \omega-\xi_{\mathbf{k}+\mathbf{Q}}$. In the temperature regime where hot spots

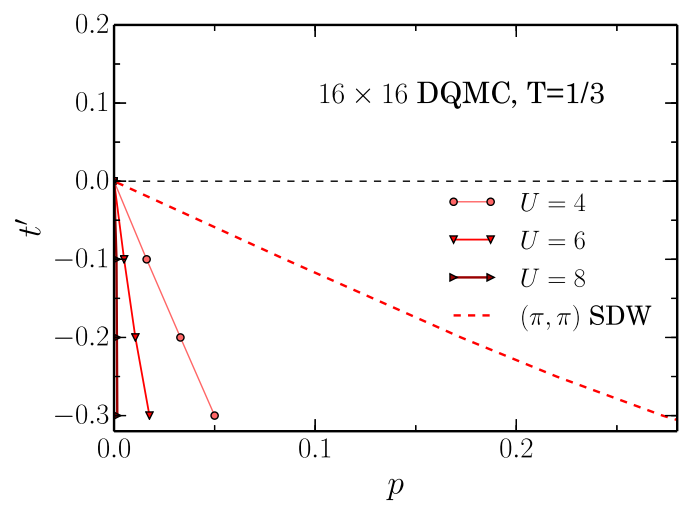

FIG. 14. The lines in the $t^{\prime}$ - $p$ plane show where the low-energy imaginary part of the antinodal self-energy $\operatorname{Im} \Sigma_{(\pi, 0)}(\omega)$ has its polelike feature centered around $\omega=0$ and an essentially particle-hole symmetric low-energy spectrum. On these curves, $\operatorname{Re} \Sigma_{(\pi, 0)}(\omega=0)$ vanishes. The solid lines are obtained by DQMC for different values of $U$, while the dashed line is the result from the SDW weak-coupling approach. The dashed line coincides with the noninteracting Lifshitz transition.

are present, $T>v_{F} / \xi$, one can distinguish two regimes of frequencies. For $\Omega_{k}>v_{F} / \xi, \operatorname{Re} \Sigma$ is of order $\left(T / \Omega_{k}\right) \ln \left(\Omega_{k} \xi / v_{F}\right)$, while at low frequencies, $\Omega_{k}<v_{F} / \xi$, the self-energy is regular, $\operatorname{Re} \Sigma \propto T \Omega_{k}\left(\xi / v_{F}\right)^{2}$. Hence, the self-energy is regular at low frequency even close to the hot spots when $\xi$ remains finite. As a result, hot spots exist at the intersection between the Fermi surface and the antiferromagnetic Brillouin zone for $T>v_{F} / \xi$, but there is no reconstruction of the Fermi surface otherwise. Hence, for a doping value larger than the value corresponding to the noninteracting Lifshitz transition, there are no hot spots and the Fermi surface is weakly renormalized and electronlike. Hence, in weak coupling, the noninteracting Lifshitz transition controls both the location of the self-energy singularities and the topological transition of the Fermi surface. This is very different from our results in the strongcoupling regime $U=7$, where these phenomena are controlled by three different lines.

By varying $U$, one can observe how the transition from weak to strong coupling happens. Figure 14 shows the DQMC results for several values of $U$. The lines show where the real part of the self-energy vanishes. It separates a region where the pole in the self-energy is at negative energies and one where it is on the positive side. It is seen that, as $U$ becomes smaller, the lines slowly approach the noninteracting Lifshitz transition, as expected in weak coupling.

\section{APPENDIX F: COMPARISON OF THE CHARGON AND ELECTRON SELF-ENERGY IN THE SU(2) GAUGE THEORY}

Here, we illustrate in more detail the role of the convolution that allows us to recover the electronic 

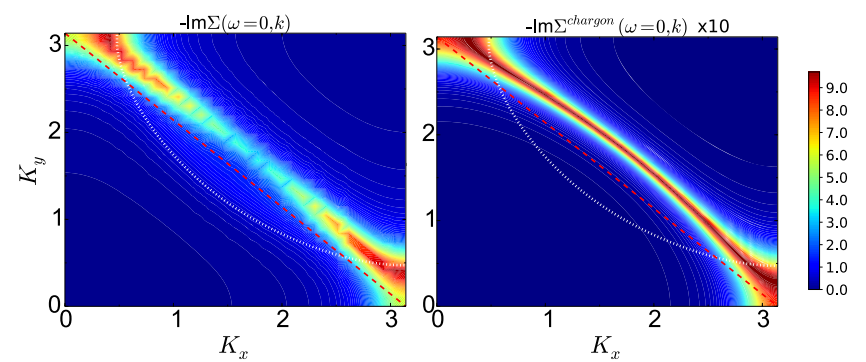

FIG. 15. Scattering rate of the electrons $-\operatorname{Im} \Sigma_{k}(\omega=0)$ and chargons $-\operatorname{Im} \Sigma_{k}^{\text {chargon }}(\omega=0)$ in momentum space. The parameters used here are $H_{0}=0.2, J=0.1 ; T=1 / 30, t^{\prime}=-0.3$, $Z_{t}=0.31, Z_{t^{\prime}}=0.19, \Delta=0.01, p=0.05$. Left panel: The physical electron self-energy has clear nodal/antinodal differentiation with a stronger scattering at the antinode than at the node. Right panel: The chargon self-energy is given by Eq. (8) and has no momentum differentiation. The broadening in the chargon self-energy is $\eta=0.04$.

Green's function in the SU(2) theory. As we have discussed above (see, e.g., Fig. 6), the location in momentum and frequency of the most singular structures of the physical self-energy is not affected by the convolution and they are already encoded in the chargon self-energy given by Eq. (8). The convolution mainly smears $G_{\psi}$ and the electron self-energy is a broadened counterpart of the chargon self-energy. A more detailed inspection shows that the convolution also redistributes spectral weight over the Brillouin zone. As a result, the physical electron selfenergy displays nodal/antinodal differentiation, which is absent in the chargon self-energy. This is illustrated in Fig. 15 where it is clearly seen that the imaginary part of the electronic self-energy is larger close to the antinode than at the node. This differentiation is not present in the chargon self-energy.

[1] M. R. Norman, D. Pines, and C. Kallin, The Pseudogap: Friend or Foe of High Tc?, Adv. Phys. 54, 715 (2005).

[2] H. Alloul, What Is the Simplest Model That Captures the Basic Experimental Facts of the Physics of Underdoped Cuprates?, C.R. Phys. 15, 519 (2014).

[3] S. Benhabib, A. Sacuto, M. Civelli, I. Paul, M. Cazayous, Y. Gallais, M.-A. Méasson, R. D. Zhong, J. Schneeloch, G. D. $\mathrm{Gu}, \mathrm{D}$. Colson, and A. Forget, Collapse of the Normal-State Pseudogap at a Lifshitz. Transition in the $\mathrm{Bi}_{2} \mathrm{Sr}_{2} \mathrm{CaCu}_{2} \mathrm{O}_{8+\delta}$ Cuprate Superconductor, Phys. Rev. Lett. 114, 147001 (2015).

[4] B. Loret, S. Sakai, S. Benhabib, Y. Gallais, M. Cazayous, M. A. Méasson, R. D. Zhong, J. Schneeloch, G. D. Gu, A. Forget, D. Colson, I. Paul, M. Civelli, and A. Sacuto, Vertical Temperature Boundary of the Pseudogap under the Superconducting Dome in the Phase Diagram of $\mathrm{Bi}_{2} \mathrm{Sr}_{2} \mathrm{CaCu}_{2} \mathrm{O}_{8+}$, Phys. Rev. B 96, 094525 (2017).

[5] N. Doiron-Leyraud, O. Cyr-Choiniére, S. Badoux, A. Ataei, C. Collignon, A. Gourgout, S. Dufour-Beauseéjour,
F. F. Tafti, F. Laliberté, M. Matusiak, D. Graf, M. Kim, J.-S. Zhou, N. Momono, T. Kurosawa, H. Takagi, and L. Taillefer, Pseudogap Phase of Cuprate Superconductors Confined by Fermi Surface Topology, Nat. Commun. 8, 2044 (2017).

[6] C. Honerkamp, M. Salmhofer, N. Furukawa, and T. M. Rice, Breakdown of the Landau-Fermi Liquid in Two Dimensions Due to Umklapp Scattering, Phys. Rev. B 63, 035109 (2001).

[7] Ar. Abanov, A. V. Chubukov, and J. Schmalian, QuantumCritical Theory of the Spin-Fermion Model and Its Application to Cuprates: Normal State Analysis, Adv. Phys. 52, 119 (2003).

[8] H. v. Löhneysen, A. Rosch, M. Vojta, and P. Wölfle, FermiLiquid Instabilities at Magnetic Quantum Phase Transitions, Rev. Mod. Phys. 79, 1015 (2007).

[9] A.-M. S. Tremblay, B. Kyung, and D. Sénéchal, Pseudogap and High-Temperature Superconductivity from Weak to Strong Coupling: Towards a Quantitative Theory, Low Temp. Phys. 32, 424 (2006).

[10] M. A. Metlitski and S. Sachdev, Quantum Phase Transitions of Metals in Two Spatial Dimensions. II. Spin Density Wave Order, Phys. Rev. B 82, 075128 (2010).

[11] X. Montiel, T. Kloss, and C. Pépin, Effective SU(2) Theory for the Pseudogap State, Phys. Rev. B 95, 104510 (2017).

[12] E. Dagotto, F. Ortolani, and D. Scalapino, Single-Particle Spectral Weight of a Two-Dimensional Hubbard Model, Phys. Rev. B 46, 3183 (1992).

[13] R. Preuss, W. Hanke, C. Gröber, and H. G. Evertz, Pseudogaps and Their Interplay with Magnetic Excitations in the Doped 2D Hubbard Model, Phys. Rev. Lett. 79, 1122 (1997).

[14] A. I. Lichtenstein and M. I. Katsnelson, Antiferromagnetism and D-Wave Superconductivity in Cuprates: A Cluster Dynamical Mean-Field Theory, Phys. Rev. B 62, R9283 (2000).

[15] T. Maier, M. Jarrell, T. Pruschke, and M. H. Hettler, Quantum Cluster Theories, Rev. Mod. Phys. 77, 1027 (2005).

[16] M. Civelli, M. Capone, S. S. Kancharla, O. Parcollet, and G. Kotliar, Dynamical Breakup of the Fermi Surface in a Doped Mott Insulator, Phys. Rev. Lett. 95, 106402 (2005).

[17] A. Macridin, M. Jarrell, T. Maier, P. R. C. Kent, and E. D'Azevedo, Pseudogap and Antiferromagnetic Correlations in the Hubbard Model, Phys. Rev. Lett. 97, 036401 (2006).

[18] B. Kyung, S. S. Kancharla, D. Sénéchal, A.-M. S. Tremblay, M. Civelli, and G. Kotliar, Pseudogap Induced by ShortRange Spin Correlations in a Doped Mott Insulator, Phys. Rev. B 73, 165114 (2006).

[19] D. J. Scalapino, Numerical Studies of the 2D Hubbard Model, in Handbook of High-Temperature Superconductivity: Theory and Experiment, edited by J. R. Schrieffer and James S. Brooks (Springer, New York, 2007), pp. 495-526.

[20] K. Haule and G. Kotliar, Strongly Correlated Superconductivity: A Plaquette Dynamical Mean-Field Theory study, Phys. Rev. B 76, 104509 (2007).

[21] M. Ferrero, P. S. Cornaglia, L. De Leo, O. Parcollet, G. Kotliar, and A. Georges, Pseudogap Opening and Formation of Fermi Arcs as an Orbital-Selective Mott Transition in Momentum Space, Phys. Rev. B 80, 064501 (2009). 
[22] E. Gull, O. Parcollet, P. Werner, and A. J. Millis, Momentum-Sector-Selective Metal-Insulator Transition in the Eight-Site Dynamical Mean-Field Approximation to the Hubbard Model in Two Dimensions, Phys. Rev. B 80, 245102 (2009).

[23] E. Gull, M. Ferrero, O. Parcollet, A. Georges, and A. J. Millis, Momentum-Space Anisotropy and Pseudogaps: A Comparative Cluster Dynamical Mean-Field Analysis of the Doping-Driven Metal-Insulator Transition in the TwoDimensional Hubbard Model, Phys. Rev. B 82, 155101 (2010).

[24] G. Sordi, K. Haule, and A.-M. S. Tremblay, Mott Physics and First-Order Transition between Two Metals in the Normal-State Phase Diagram of the Two-Dimensional Hubbard Model, Phys. Rev. B 84, 075161 (2011).

[25] G. Sordi, P. Sémon, K. Haule, and A.-M. S. Tremblay, Strong Coupling Superconductivity, Pseudogap, and Mott Transition, Phys. Rev. Lett. 108, 216401 (2012).

[26] O. Gunnarsson, T. Schäfer, J. P. F. LeBlanc, E. Gull, J. Merino, G. Sangiovanni, G. Rohringer, and A. Toschi, Fluctuation Diagnostics of the Electron Self-Energy: Origin of the Pseudogap Physics, Phys. Rev. Lett. 114, 236402 (2015).

[27] L. Fratino, P. Sémon, G. Sordi, and A.-M. S. Tremblay, Pseudogap and Superconductivity in Two-Dimensional Doped Charge-Transfer Insulators, Phys. Rev. B 93, 245147 (2016).

[28] W. Wu, M. Ferrero, A. Georges, and E. Kozik, Controlling Feynman Diagrammatic Expansions: Physical Nature of the Pseudogap in the Two-Dimensional Hubbard Model, Phys. Rev. B 96, 041105(R) (2017).

[29] M. A. Kastner, R. J. Birgeneau, G. Shirane, and Y. Endoh, Magnetic, Transport, and Optical Properties of Monolayer Copper Oxides, Rev. Mod. Phys. 70, 897 (1998).

[30] Th. A. Maier, Th. Pruschke, and M. Jarrell, Angle-Resolved Photoemission Spectra of the Hubbard Model, Phys. Rev. B 66, 075102 (2002).

[31] K.-S. Chen, Z. Y. Meng, T. Pruschke, J. Moreno, and M. Jarrell, Lifshitz Transition in the Two-Dimensional Hubbard Model, Phys. Rev. B 86, 165136 (2012).

[32] S. Sakai, Y. Motome, and M. Imada, Evolution of Electronic Structure of Doped Mott Insulators: Reconstruction of Poles and Zeros of Green's Function, Phys. Rev. Lett. 102, 056404 (2009).

[33] L. F. Tocchio, F. Becca, and C. Gros, Strong Renormalization of the Fermi-Surface Topology Close to the Mott Transition, Phys. Rev. B 86, 035102 (2012).

[34] T. D. Stanescu and G. Kotliar, Fermi Arcs and Hidden Zeros of the Green Function in the Pseudogap State, Phys. Rev. B 74, 125110 (2006).

[35] C. Berthod, T. Giamarchi, S. Biermann, and A. Georges, Breakup of the Fermi Surface near the Mott Transition in Low-Dimensional Systems, Phys. Rev. Lett. 97, 136401 (2006).

[36] N. Lin, E. Gull, and A. J. Millis, Physics of the Pseudogap in Eight-Site Cluster Dynamical Mean-Field Theory: Photoemission, Raman Scattering, and In-Plane and c-axis Conductivity, Phys. Rev. B 82, 045104 (2010).
[37] S. Sachdev, M. A. Metlitski, Y. Qi, and C. Xu, Fluctuating Spin Density Waves in Metals, Phys. Rev. B 80, 155129 (2009).

[38] S. Chatterjee, S. Sachdev, and M. Scheurer, Intertwining Topological Order and Broken Symmetry in a Theory of Fluctuating Spin Density Waves, Phys. Rev. Lett. 119, 227002 (2017).

[39] M. S. Scheurer, S. Chatterjee, W. Wu, M. Ferrero, A. Georges, and S. Sachdev, Topological Order in the Pseudogap Metal, Proc. Natl. Acad. Sci. U.S.A. 115, E3665 (2018).

[40] R. Blankenbecler, D. J. Scalapino, and R. L. Sugar, Monte Carlo Calculations of Coupled Boson-Fermion Systems. I, Phys. Rev. D 24, 2278 (1981).

[41] M. Jarrell, Th. Maier, M. H. Hettler, and A. N. Tahvildarzadeh, Phase Diagram of the Hubbard Model: Beyond the Dynamical Mean Field, Europhys. Lett. 56, 563 (2001).

[42] P. Werner, E. Gull, O. Parcollet, and A. J. Millis, Momentum-Selective Metal-Insulator Transition in the Two-Dimensional Hubbard Model: An 8-Site Dynamical Cluster Approximation Study, Phys. Rev. B 80, 045120 (2009).

[43] E. Gull, O. Parcollet, and A. J. Millis, Superconductivity and the Pseudogap in the Two-Dimensional Hubbard Model, Phys. Rev. Lett. 110, 216405 (2013).

[44] A. Damascelli, Z. Hussain, and Z.-X. Shen, Angle-Resolved Photoemission Studies of the Cuprate Superconductors, Rev. Mod. Phys. 75, 473 (2003).

[45] B. I. Shraiman and E. D. Siggia, Mobile Vacancies in a Quantum Heisenberg Antiferromagnet, Phys. Rev. Lett. 61, 467 (1988).

[46] H. J. Schulz, Effective Action for Strongly Correlated Fermions from Functional Integrals, Phys. Rev. Lett. 65, 2462 (1990).

[47] J. R. Schrieffer, Pairing, Magnetic Spin Fluctuations, and Superconductivity near a Quantum Critical Point, J. Supercond. 17, 539 (2004).

[48] E. Fradkin and S. H. Shenker, Phase Diagrams of Lattice Gauge Theories with Higgs Fields, Phys. Rev. D 19, 3682 (1979).

[49] N. Read and S. Sachdev, Large N Expansion for Frustrated Quantum Antiferromagnets, Phys. Rev. Lett. 66, 1773 (1991).

[50] X. G. Wen, Mean-Field Theory of Spin-Liquid States with Finite Energy Gap and Topological Orders, Phys. Rev. B 44, 2664 (1991).

[51] F. A. Bais, P. van Driel, and M. de Wild Propitius, Quantum Symmetries in Discrete Gauge Theories, Phys. Lett. B 280 , 63 (1992).

[52] T. Senthil, S. Sachdev, and M. Vojta, Fractionalized Fermi Liquids, Phys. Rev. Lett. 90, 216403 (2003).

[53] K.-Y. Yang, T. M. Rice, and F.-C. Zhang, Phenomenological Theory of the Pseudogap State, Phys. Rev. B 73, 174501 (2006).

[54] S. Sakai, M. Civelli, and M. Imada, Hidden Fermionic Excitation Boosting High-Temperature Superconductivity in Cuprates, Phys. Rev. Lett. 116, 057003 (2016).

[55] K. B. Dave, P. W. Phillips, and C. L. Kane, Absence of Luttinger's Theorem Due to Zeros in the Single-Particle Green Function, Phys. Rev. Lett. 110, 090403 (2013). 
[56] Y. Qi and S. Sachdev, Effective Theory of Fermi Pockets in Fluctuating Antiferromagnets, Phys. Rev. B 81, 115129 (2010).

[57] E. Pavarini, I. Dasgupta, T. Saha-Dasgupta, O. Jepsen, and O. K. Andersen, Band-Structure Trend in Hole-Doped Cuprates and Correlation with $T_{c \max }$, Phys. Rev. Lett. 87, 047003 (2001).

[58] R. A. Cooper, Y. Wang, B. Vignolle, O. J. Lipscombe, S. M. Hayden, Y. Tanabe, T. Adachi, Y. Koike, M. Nohara, H. Takagi et al., Anomalous Criticality in the Electrical Resistivity of $\mathrm{La}_{2-x} \mathrm{Sr}_{x} \mathrm{CuO}_{4}$, Science 323, 603 (2009).

[59] J. Chang, M. Shi, S. Pailhés, M. Månsson, T. Claesson, O. Tjernberg, A. Bendounan, Y. Sassa, L. Patthey, N. Momono et al., Anisotropic Quasiparticle Scattering Rates in Slightly Underdoped to Optimally Doped High-Temperature $\mathrm{La}_{2-x} \mathrm{Sr}_{x} \mathrm{CuO}_{4}$ Superconductors, Phys. Rev. B 78, 205103 (2008).

[60] T. Yoshida, X. J. Zhou, K. Tanaka, W. L. Yang, Z. Hussain, Z.-X. Shen, A. Fujimori, S. Sahrakorpi, M. Lindroos, R. S. Markiewicz et al., Systematic Doping Evolution of the Underlying Fermi Surface of $\mathrm{La}_{2-x} \mathrm{Sr}_{x} \mathrm{CuO}_{4}$, Phys. Rev. B 74, 224510 (2006).

[61] S. R. Park, Y. Cao, Q. Wang, M. Fujita, K. Yamada, S.-K. Mo, D. S. Dessau, and D. Reznik, Broken Relationship between Superconducting Pairing Interaction and Electronic Dispersion Kinks in $\mathrm{La}_{2-x} \mathrm{Sr}_{x} \mathrm{CuO}_{4}$ Measured by Angle-Resolved Photoemission, Phys. Rev. B 88, 220503 (2013).

[62] C. Collignon, S. Badoux, S. A. A. Afshar, B. Michon, F. Laliberte, O. Cyr-Choiniere, J.-S. Zhou, S. Licciardello, S. Wiedmann, N. Doiron-Leyraud, and L. Taillefer, FermiSurface Transformation across the Pseudogap Critical Point of the Cuprate Superconductor $\mathrm{La}_{1.6-x} \mathrm{Nd}_{0.4} \mathrm{Sr}_{x} \mathrm{CuO}_{4}$, Phys. Rev. B 95, 224517 (2017).

[63] C. E. Matt et al., Electron Scattering, Charge Order, and Pseudogap Physics in $\mathrm{La}_{1.6-x} \mathrm{Nd}_{0.4} \mathrm{Sr}_{x} \mathrm{CuO}_{4}$ : An AngleResolved Photoemission Spectroscopy Study, Phys. Rev. B 92, 134524 (2015).

[64] Y. He, Y. Yin, M. Zech, A. Soumyanarayanan, M. M. Yee, T. Williams, M. C. Boyer, K. Chatterjee, W. D. Wise, I. Zeljkovic et al., Fermi Surface and Pseudogap Evolution in a Cuprate Superconductor, Science 344, 608 (2014).

[65] G.-q. Zheng, P. L. Kuhns, A. P. Reyes, B. Liang, and C. T. Lin, Critical Point and the Nature of the Pseudogap of Single-Layered Copper-Oxide $\mathrm{Bi}_{2} \mathrm{Sr}_{2-x} \mathrm{La}_{x} \mathrm{CuO}_{6+\delta}$ Superconductors, Phys. Rev. Lett. 94, 047006 (2005).

[66] S. Kawasaki, C. Lin, P. L. Kuhns, A. P. Reyes, and G.-q. Zheng, Carrier-Concentration Dependence of the Pseudogap Ground State of Superconducting $\mathrm{Bi}_{2} \mathrm{Sr}_{2-x} \mathrm{La}_{x} \mathrm{CuO}_{6+\delta}$ Revealed by ${ }^{63,65} \mathrm{Cu}-$ Nuclear Magnetic Resonance in Very High Magnetic Fields, Phys. Rev. Lett. 105, 137002 (2010).

[67] T. Kondo, T. Takeuchi, T. Yokoya, S. Tsuda, S. Shin, and U. Mizutani, Hole-Concentration Dependence of Band Structure in $(\mathrm{Bi}, \mathrm{Pb})_{2}(\mathrm{Sr}, \mathrm{La})_{2} \mathrm{CuO}_{6+\delta}$ Determined by the Angle-Resolved Photoemission Spectroscopy, J. Electron Spectrosc. Relat. Phenom. 137-140, 663 (2004).

[68] A. Kaminski, S. Rosenkranz, H. M. Fretwell, M. R. Norman, M. Randeria, J. C. Campuzano, J. M. Park, Z. Z. Li, and H. Raffy, Change of Fermi-Surface Topology in
$\mathrm{Bi}_{2} \mathrm{Sr}_{2} \mathrm{CaCuO}_{2} \mathrm{O}_{8+\delta}$ with Doping, Phys. Rev. B 73, 174511 (2006).

[69] M. A. Hossain, J. D. F. Mottershead, D. Fournier, A. Bostwick, J. L. McChesney, E. Rotenberg, R. Liang, W. N. Hardy, G. A. Sawatzky, I. S. Elfimov et al., In Situ Doping Control of the Surface of High-Temperature Superconductors, Nat. Phys. 4, 527 (2008).

[70] S. Badoux, W. Tabis, F. Laliberté, G. Grissonnanche, B. Vignolle, D. Vignolles, J. Béard, D. A. Bonn, W. N. Hardy, R. Liang et al., Change of Carrier Density at the Pseudogap Critical Point of a Cuprate Superconductor, Nature (London) 531, 210 (2016).

[71] M. Platé, J. D. F. Mottershead, I. S. Elfimov, D. C. Peets, Ruixing Liang, D. A. Bonn, W. N. Hardy, S. Chiuzbaian, M. Falub, M. Shi et al., Fermi Surface and Quasiparticle Excitations of Overdoped $\mathrm{Tl}_{2} \mathrm{Ba}_{2} \mathrm{CuO}_{6+\delta}$, Phys. Rev. Lett. 95, 077001 (2005).

[72] C. Proust, E. Boaknin, R. W. Hill, L. Taillefer, and A. P. Mackenzie, Heat Transport in a Strongly Overdoped Cuprate: Fermi Liquid and a Pure D-wave BCS Superconductor, Phys. Rev. Lett. 89, 147003 (2002).

[73] W. Tabis, Y. Li, M. Le Tacon, L. Braicovich, A. Kreyssig, M. Minola, G. Dellea, E. Weschke, M. J. Veit, M. Ramazanoglu et al., Charge Order and Its Connection with Fermi-Liquid Charge Transport in a Pristine High-Tc Cuprate, Nat. Commun. 5, 5875 (2014).

[74] Y. Kohsaka, C. Taylor, K. Fujita, A. Schmidt, C. Lupien, T. Hanaguri, M. Azuma, M. Takano, H. Eisaki, H. Takagi et al., An Intrinsic Bond-Centered Electronic Glass with Unidirectional Domains in Underdoped Cuprates, Science 315, 1380 (2007).

[75] Ø. Fischer, M. Kugler, I. Maggio-Aprile, C. Berthod, and C. Renner, Scanning Tunneling Spectroscopy of HighTemperature Superconductors, Rev. Mod. Phys. 79, 353 (2007).

[76] S. Sakai, S. Blanc, M. Civelli, Y. Gallais, M. Cazayous, M.-A. Méasson, J. S. Wen, Z. J. Xu, G. D. Gu, G. Sangiovanni, Y. Motome, K. Held, A. Sacuto, A. Georges, and M. Imada, Raman-Scattering Measurements and Theory of the Energy-Momentum Spectrum for Underdoped $\mathrm{Bi}_{2} \mathrm{Sr}_{2} \mathrm{CaCu}_{8+\delta}$ Superconductors: Evidence of an s-wave Structure for the Pseudogap, Phys. Rev. Lett. 111, 107001 (2013).

[77] J. E. Hirsch and R. M. Fye, Monte Carlo Method for Magnetic Impurities in Metals, Phys. Rev. Lett. 56, 2521 (1986).

[78] A. Georges, G. Kotliar, W. Krauth, and M. J. Rozenberg, Dynamical Mean-Field Theory of Strongly Correlated Fermion Systems and the Limit of Infinite Dimensions, Rev. Mod. Phys. 68, 13 (1996).

[79] A. N. Rubtsov, V. V. Savkin, and A. I. Lichtenstein, Continuous-Time Quantum Monte Carlo Method for Fermions, Phys. Rev. B 72, 035122 (2005).

[80] D. Bergeron and A.-M.S. Tremblay, Algorithms for Optimized Maximum Entropy and Diagnostic Tools for Analytic Continuation, Phys. Rev. E 94, 023303 (2016).

[81] M. Jarrell and J. E. Gubernatis, Bayesian Inference and the Analytic Continuation of Imaginary-Time Quantum Monte Carlo Data, Phys. Rep. 269, 133 (1996). 
[82] N. Trivedi and M. Randeria, Deviations from Fermi-Liquid Behavior above $T_{c}$ in 2D Short Coherence Length Superconductors, Phys. Rev. Lett. 75, 312 (1995).

[83] X. Deng, J. Mravlje, R. Žitko, M. Ferrero, G. Kotliar, and A. Georges, How Bad Metals Turn Good: Spectroscopic Signatures of Resilient Quasiparticles, Phys. Rev. Lett. 110, 086401 (2013).

[84] B. S. Shastry, Extremely Correlated Fermi Liquids, Phys. Rev. Lett. 107, 056403 (2011).

[85] Y. M. Vilk and A.-M.S. Tremblay, Non-perturbative Many-Body Approach to the Hubbard Model and
Single-Particle Pseudogap, J. Phys. I (France) 7, 1309 (1997).

[86] A.-M. S. Tremblay, Two-Particle-Self-Consistent Approach for the Hubbard Model, in Strongly Correlated Systems: Theoretical Methods, edited by A. Avella and F. Mancini (Springer, Berlin, Heidelberg, 2012), pp. 409-453.

Correction: Missing information in Ref. [5] has been inserted. 